\title{
Moret-Bailly families and non-liftable schemes
}

\author{
Damian Rössler and Stefan Schröer
}

\begin{abstract}
Generalizing the Moret-Bailly pencil of supersingular abelian surfaces to higher dimensions, we construct for each field of characteristic $p>0$ a smooth projective variety with trivial dualizing sheaf that does not lift to characteristic zero. Our approach heavily relies on local unipotent group schemes, the Beauville-Bogomolov decomposition for Kähler manifolds with $c_{1}=0$, and equivariant deformation theory in mixed characteristics.
\end{abstract}

\section{Introduction}

Every compact Kähler manifold $V$ with Chern class $c_{1}=0$ has unobstructed deformations, although the obstruction group $H^{2}\left(V, \Theta_{V}\right)$ is usually non-zero. This foundational fact relies on the $T^{1}$-lifting theorem (cf. [Bog78, Tod80, Tia87, Kaw92, Ran92]). It holds, in particular, for complex tori, hyperkähler manifolds, and Calabi-Yau manifolds. In fact, the Beauville-Bogomolov decomposition theorem asserts that a compact Kähler manifold $V$ with $c_{1}=0$ admits a finite étale covering $V^{\prime} \rightarrow V$ that splits into a product $V^{\prime}=V_{1} \times \cdots \times V_{r}$, where the factors belong to these three classes [Bog74, Bea83].

Over fields $k$ of characteristic $p>0$, much less is known for smooth proper schemes $Y$ that have $c_{1}=0$, in the sense that the dualizing sheaf $\omega_{Y}$ is numerically trivial. Again we have three particular classes: The abelian varieties take over the role of complex tori. Copying the definition in characteristic zero, one may call $Y$ a hyperkähler manifold if it is simply connected and there is some $\sigma \in H^{0}\left(Y, \Omega_{Y}^{2}\right)$ whose adjoint map $\sigma: \Theta_{Y} \rightarrow \Omega_{Y / k}^{1}$ is bijective. Similarly, $Y$ is a Calabi-Yau manifold if it is simply connected with $h^{i}\left(\mathscr{O}_{Y}\right)=0$ for $1<i<\operatorname{dim}(Y)$.

Under strong additional assumptions, analogs of the $T^{1}$-lifting theorem [ES05, Sch03] and the decomposition theorem [PZ19] hold true. In light of the liftability of abelian varieties [Kat81, NO80] and K3-surfaces [Del81], it is natural to wonder whether any such $Y$ admits a lifting to characteristic zero. This, however, turns out to be false already for Calabi-Yau threefolds. The first example was given by Hirokado [Hir99] in characteristic $p=3$. The second author [Sch04] found further examples in characteristic $p=2,3$ using quotients of the Moret-Bailly pencil of supersingular abelian surfaces [Mor81]. Further examples in dimension three at certain bounded sets of primes were constructed by Schoen [Sch09], Hirokado, Ito, and Saito [HIS07, HIS08],

Received 4 August 2020, accepted in final form 4 July 2021.

2020 Mathematics Subject Classification 14J32, 14K05, 14L15, $14 \mathrm{D} 10$.

Keywords: Moret-Bailly family, abelian varieties, group schemes, Calabi-Yau manifolds.

This journal is (C) Foundation Compositio Mathematica 2022. This article is distributed with Open Access under the terms of the Creative Commons Attribution Non-Commercial License, which permits non-commercial reuse, distribution, and reproduction in any medium, provided that the original work is properly cited. For commercial re-use, please contact the Foundation Compositio Mathematica.

The research was supported by the Deutsche Forschungsgemeinschaft via the grant SCHR 671/6-1 EnriquesMannigfaltigkeiten. It was also conducted in the framework of the research training group GRK 2240: Algebrogeometric Methods in Algebra, Arithmetic and Topology. 


\section{RÖSSLER AND S. SCHRÖER}

Cynk and Van Straten [CvS09], and Cynk and Schütt [CS12]. Finally, Achinger and Zdanowicz [AZ17, Zda21] produced for each prime $p \geqslant 5$ a non-liftable Calabi-Yau manifold of dimension 2p, based on the failure of Kodaira vanishing as observed by Totaro [Tot19].

The goal of this paper is to generalize the Moret-Bailly pencil to higher dimensions and establish further non-liftability results: Let $A=E_{1} \times \cdots \times E_{g}$ be a product of supersingular elliptic curves. Roughly speaking, the embeddings of the local unipotent group scheme $\alpha_{p}=\mathbb{G}_{a}[F]$ into the abelian variety $A$ are parameterized by the projectivization $\mathbb{P}^{n}=\mathbb{P}(\mathfrak{a})$ of the Lie algebra $\mathfrak{a}=\operatorname{Lie}(A)$, where $n+1=g$. In fact, any inclusion $\mathscr{O}_{\mathbb{P} n}(-d) \subset \mathscr{O}_{\mathbb{P} n}^{\oplus(n+1)}$ that is locally a direct summand corresponds to a family $H \subset A \times \mathbb{P}^{n}$ of such finite local group schemes. Setting $X=A \times \mathbb{P}^{n}$, we then form the family of quotients $Y=X / H$, which comes with a radical surjection $\epsilon: X \rightarrow Y$ and an induced fibration $\varphi: Y \rightarrow \mathbb{P}^{n}$.

We call the fibrations $\varphi: Y \rightarrow \mathbb{P}^{n}$ and also the smooth proper schemes $Y$ Moret-Bailly families since they are higher-dimensional analogs of the famous construction of a non-isotrivial family of abelian surfaces over the projective line [Mor81]; such families were already mentioned by Grothendieck in [Gro66, Remark 4.6].

The dimension of $Y$ is $2 n+1=2 g-1$. It is easy to compute the Betti numbers $b_{i}(Y)$, but the cohomological invariants $h^{i}\left(\mathscr{O}_{Y}\right)$ remain mysterious. Using a result of Achinger [Ach15] on the splitting type of the Frobenius push-forward on toric varieties, one may express $h^{i}\left(\mathscr{O}_{Y}\right)$ via lattice point counts. It turns out that the canonical projection $\psi: Y \rightarrow A^{(p)}=A / A[F]$ is the Albanese map, and the dualizing sheaf $\omega_{Y}$ is the pullback of $\mathscr{O}_{\mathbb{P}^{n}}(m)$ for the integer $m=d(p-1)-g$. In particular, we have $c_{1}=0$ if and only if $d(p-1)=g$, and in this case $\omega_{Y}=\mathscr{O}_{Y}$. More generally, $\omega_{Y}$ is anti-nef, which means that $\left(\omega_{Y} \cdot C\right) \leqslant 0$ for every integral curve $C \subset Y$, if and only if $d(p-1) \leqslant g$. The first main result of this paper is the following.

Theorem A (See Theorem 5.1). Suppose $d(p-1) \leqslant g$ and $p \geqslant 3$. Then the Moret-Bailly family $Y$ does not projectively lift to characteristic zero.

In the boundary case $d(p-1)=g$, this apparently gives the first examples of non-liftable manifolds with $c_{1}=0$ that do not belong to the class of abelian varieties, hyperkähler manifolds, Calabi-Yau manifolds, or products thereof. To establish the result, we assume that a projective lifting $\mathfrak{Y} \rightarrow \operatorname{Spec}(R)$ exists and use the existence of relative Hilbert schemes Hilb $_{\mathfrak{Y} / R}$ to show that properties of the Albanese map $V \rightarrow \mathrm{Alb}_{V / \mathbb{C}}$ for the resulting complex fiber $V=\mathfrak{Y} \otimes_{R} \mathbb{C}$ would contradict a recent result of Cao [Cao19]. In the case where $d(p-1)=g$, the contradiction could also be derived from the Beauville-Bogomolov decomposition theorem for Kähler manifolds with $c_{1}=0 .^{1}$

We strongly believe that Moret-Bailly families do not even formally lift to characteristic zero. So far we are not able to show this, but we can prove the following, which takes into account the sign involution on $Y$, viewed as a family of abelian varieties parameterized by $\mathbb{P}^{n}$.

Theorem B (See Theorem 8.2). Suppose $d=1, p-1 \leqslant g$, and $p \geqslant 3$. Then $Y$ together with its sign involution does not formally lift to characteristic zero.

We show that any formal lifting $\mathfrak{Y}$ to characteristic zero, in which the sign involution extends, admits an ample sheaf. Then Grothendieck's existence theorem gives a contradiction

\footnotetext{
${ }^{1}$ In a first version of this paper, we considered only the case $d(p-1)=g$. Ludvig Olsson discovered that our result could be extended to the situation where the dual of $\omega_{Y}$ is nef, if one avails oneself of the result of Cao. We are very grateful to him for sharing his insight with us and allowing us to make use of it in this paper.
} 


\section{Moret-BAILly FAMILIES AND NON-LIFTABLE SCHEMES}

to Theorem A. This argument relies on Rim's equivariant deformation theory [Rim80] and its generalization to mixed characteristics [ST18] and on a computation of weights in the groups $H^{2}\left(Y, \mathscr{O}_{Y}\right)$ and $H^{1}\left(Y, \Theta_{Y}\right)$ for the action of $G=\{ \pm 1\}$ coming from the sign involution on $A$. In the first version of the article, we overlooked some contribution to the weights, which led to an unjustified stronger assertion. It turns out that extending the sign involution to infinitesimal deformations $\mathfrak{Y}$ of $Y$ is actually the same as extending the morphism $\varphi: Y \rightarrow \mathbb{P}^{n}$ (see Proposition 8.4).

In the last section of this article, we consider the possibility of lifting Moret-Bailly families to the ring $W_{2}(k)$ of Witt vectors of length two. The third main result is the following.

Theorem C (See Theorem 9.1). Fix $n \geqslant 2$ and $d \geqslant 1$. If $n \not \equiv 2$ modulo 4 and the ground field $k$ is perfect, then the Moret-Bailly family $Y$ does not lift to $W_{2}(k)$ for almost all primes $p>0$.

Note that in contrast to the previous results, here there are no assumptions on the dualizing sheaf $\omega_{Y}$. Of course there still may be deformations over some finite flat extensions of $W_{2}(k)$. In the special case $n=3$, we actually can show that $Y$ does not lift to $W_{2}(k)$ for all primes $p \geqslant 7$.

To prove Theorem C, we construct certain ample sheaves on the Moret-Bailly family $Y$ that violate the conclusion of the Kodaira vanishing theorem. By results of Deligne and Illusie [DI87], this ensures non-liftability to $W_{2}(k)$. The computation relies on the Hirzebruch-Riemann-Roch theorem, together with an analysis of the Todd class $\operatorname{td}\left(\Theta_{Y}\right)$ and properties of the Bernoulli numbers $B_{i} \in \mathbb{Q}$.

The paper is organized as follows: In Section 1, we collect some facts on families of algebraic group schemes in characteristic $p>0$ and discuss the four-term complex that describes certain infinitesimal quotients. In Section 2, we apply this to families of abelian varieties. The MoretBailly families $\varphi: Y \rightarrow \mathbb{P}^{n}$ are introduced in Section 3, where we compute the dualizing sheaf and Betti numbers. Section 4 contains a description of the Picard scheme and the Albanese map. Fibers of the Albanese map play a crucial role in Section 5, where we prove that Moret-Bailly families with $c_{1}=0$ do not projectively lift to characteristic zero. Here the main ingredients are relative Hilbert schemes and the Beauville-Bogomolov decomposition over the complex numbers. In Section 6, we express the cohomology groups $H^{i}\left(Y, \mathscr{O}_{Y}\right)$ in terms of cohomology on $\mathbb{P}^{n}$ for coefficient sheaves that involve Frobenius pullbacks and exterior powers. This is used in Section 7 to compute weights in $H^{2}\left(Y, \mathscr{O}_{Y}\right)$ and $H^{1}\left(Y, \Theta_{Y}\right)$, which are the crucial obstruction groups for infinitesimal deformations. Section 8 contains the proof that Moret-Bailly families $Y$ with $c_{1}=0$, together with the sign involution, do not admit a formal lift to characteristic zero, using equivariant deformation theory in mixed characteristics. In Section 9, we show that there are ample invertible sheaves on $Y$ which violate the conclusion of the Kodaira vanishing theorem when $n, d, p$ satisfy certain numerical conditions.

\section{Families of algebraic group schemes}

Let $S$ be a base scheme. A family of algebraic group schemes is a scheme $X$, together with a morphism $X \rightarrow S$ that is flat and of finite presentation, endowed with the structure of a relative group scheme. Here we could allow algebraic spaces as well. However, for the sake of exposition we stay in the realm of schemes, which is sufficient for our applications.

Let us assume that the sheaf of Kähler differentials $\Omega_{X / S}^{1}$ is locally free. Then the tangent sheaf $\Theta_{X / S}=\underline{\operatorname{Hom}}\left(\Omega_{X / S}^{1}, \mathscr{O}_{X}\right)$ is locally free as well. The sheaf of Lie algebras $\operatorname{Lie}_{X / S}$ is the pullback of $\Theta_{X / S}$ along the neutral section $e: S \rightarrow X$. This is a locally free sheaf, endowed with 


\section{RÖSSLER AND S. SCHRÖER}

a Lie bracket, such that the fibers $\mathfrak{g}=\operatorname{Lie}_{X / S} \otimes \kappa(a)$, for $a \in S$, become Lie algebras over the residue fields $\kappa(a)$.

Now suppose that $S$ has characteristic $p>0$. Then the sheaf of Lie algebras $\operatorname{Lie}_{X / S}$ acquires the $p$-map as additional structure, so that the $\mathfrak{g}=\operatorname{Lie}_{X / S} \otimes \kappa(a)$ become restricted Lie algebras over $\kappa(a)$. Recall that the map

$$
\operatorname{Hom}(X, Y) \longrightarrow \operatorname{Hom}\left(\operatorname{Lie}_{X / S}, \operatorname{Lie}_{Y / S}\right)
$$

is bijective provided that $X$ has height at most one, according to [DG70a, Exposé $\mathrm{VII}_{A}$, Theorem 7.2]. Note that the hom set on the right comprises $\mathscr{O}_{S}$-linear maps compatible with Lie bracket and $p$-map, and that height at most one means that the relative Frobenius $F: X \rightarrow X^{(p)}$ is trivial. Here $X^{(p)}$ is the pullback of $X$ along the absolute Frobenius map $F_{S}: S \rightarrow S$, and the morphism $F$ comes from the commutative diagram

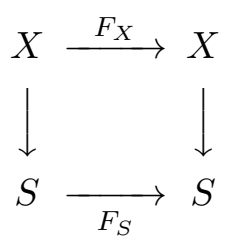

of absolute Frobenius maps. Moreover, each sheaf $\mathscr{H}$ of restricted Lie algebras that is locally free of finite rank arises from some family of algebraic group schemes $H$ of height at most one. In fact, $H$ is the relative spectrum of the sheaf of algebras

$$
\mathscr{A}=\mathscr{H} o m\left(U^{[p]}(\mathscr{H}), \mathscr{O}_{S}\right) \text {, }
$$

where $U^{[p]}(\mathscr{H})$ is the quotient of the sheaf $U(\mathscr{H})$ of universal enveloping algebras by some sheaf of ideals defined via the $p$-map, as explained in [DG70a, Exposé VII ${ }_{A}$, Section 5]; also compare with [DG70b, Chapter II, Section 7, No. 4].

Let $\mathscr{H} \subset \operatorname{Lie}_{X / S}$ be a subsheaf that is locally a direct summand, and assume that $\mathscr{H}$ is stable under both Lie bracket and $p$-map. Let $H \rightarrow S$ be the corresponding family of group schemes of height at most one, with $\operatorname{Lie}_{H / S}=\mathscr{H}$. We now consider the inclusion $H \subset X$ and form the resulting quotient $Y=X / H$. Such a quotient exists as an algebraic space. It is actually a scheme because the projection $X \rightarrow Y$ is a finite universal homeomorphism [Ols16, Theorem 6.2.2]. If $H$ is normal, $Y$ inherits the structure of a family of algebraic groups.

Proposition 1.1. The structure morphism $Y \rightarrow S$ is flat and of finite presentation. Moreover, it is smooth provided that $X \rightarrow S$ is smooth.

Proof. The projection $\epsilon: X \rightarrow Y$ is faithfully flat and of finite presentation because it is a torsor with respect to $H \times Y$. The assertion now follows from fppf descent.

In the special case $\mathscr{H}=\mathrm{Lie}_{X / S}$, the group scheme $H$ coincides with the kernel $X[F]$ of the relative Frobenius map. In the general case, we thus have an $S$-morphism $X / H \rightarrow X^{(p)}$.

We now assume $H=X[F]$ and furthermore that $X$ is smooth. Then the same holds for $Y$, and the homomorphism $X \rightarrow X^{(p)}$ is an epimorphism, so that $X / H=X^{(p)}$. In turn, we obtain an exact sequence $0 \rightarrow \mathscr{H} \rightarrow \mathrm{Lie}_{X / S} \rightarrow \mathrm{Lie}_{Y / S}$ of families of restricted Lie algebras. By assumption, the inclusion on the left is locally a direct summand, so the cokernel $\mathscr{K}=\mathrm{Lie}_{X / S} / \mathscr{H}$ is locally free. Since forming the quotient $Y=X / H$ commutes with base change, the inclusion $\mathscr{K} \subset \operatorname{Lie}_{Y / S}$ is locally a direct summand. Let $K \subset Y$ be the corresponding family of group schemes of height at most one. The isomorphism theorem ensures $Y / K=X / X[F]=X^{(p)}$. This 
gives a commutative diagram

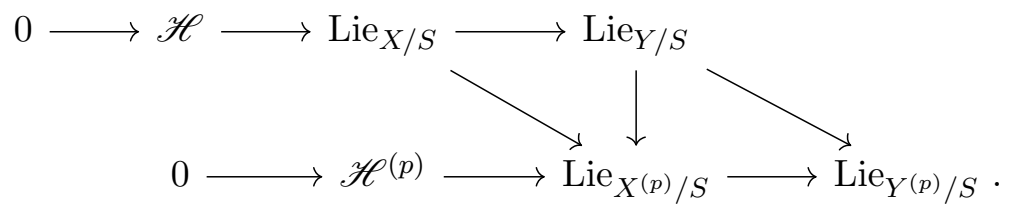

The two diagonal maps vanish, hence the vertical map factors over $\mathscr{H}^{(p)}$, and we obtain the four-term complex

$$
0 \longrightarrow \mathscr{H} \longrightarrow \operatorname{Lie}_{X / S} \longrightarrow \operatorname{Lie}_{Y / S} \longrightarrow \mathscr{H}^{(p)} \longrightarrow 0
$$

Sequences like this already appear in Ekedahl's work on foliations of smooth algebraic schemes [Eke87, Corollary 3.4].

TheOREM 1.2. The complex (1.3) of restricted Lie algebras is exact.

Proof. By construction, the complex is exact at all terms, with the possible exception of $\mathscr{H}^{(p)}$. Our task is thus to verify that $\operatorname{Lie}_{Y / S} \rightarrow \mathscr{H}^{(p)}$ is surjective. By the Nakayama lemma, it suffices to do so after tensoring with $\kappa(a)$, for $a \in S$. Since the formation of quotients commutes with base change, so does the formation of the complex. It thus suffices to treat the case where $S$ itself is the spectrum of a field. Now the terms become finite-dimensional vector spaces. The outer terms have the same dimension, and the same holds for the inner terms. In turn, the rank of $\operatorname{Lie}_{Y / S} \rightarrow \mathscr{H}^{(p)}$ coincides with the dimension of $\mathscr{H}^{(p)}$, so the map in question must be surjective.

\section{Families of abelian varieties}

We keep the assumptions of the preceding section and now assume that $\psi: X \rightarrow S$ is a family of abelian varieties of relative dimension $g \geqslant 0$. According to [Art69, Theorem 7.3], the relative Picard functor Pic $X / S$ is representable by an algebraic space, and $P=\mathrm{Pic}_{X / S}^{0}$ is called the family of dual abelian varieties. The sheaf of Lie algebras is given by $\operatorname{Lie}_{P / S}=R^{1} \psi_{*}\left(\mathscr{O}_{X}\right)$, with trivial bracket. Note that by results of Raynaud, any family of abelian varieties has schematic total space but is not necessarily projective ([FC90, Theorem 1.9] and [Ray70, Chapter VII, Section 4.2], also compare with the discussion in [LS21, Section 4]).

Let $X \rightarrow Y$ be a homomorphism between two families of abelian varieties. It must be flat, by the fiber-wise criterion for flatness [GD66, Theorem 11.3.10]. Also suppose $\operatorname{dim}\left(X_{s}\right)=\operatorname{dim}\left(Y_{s}\right)$ for all points $s \in S$. Then the kernel $H \subset X$ is a family of finite group schemes, and the Cartier dual $\operatorname{Hom}\left(H, \mathbb{G}_{m}\right)$ is another family of finite group schemes. As explained in [Oda69, Corollary 1.3], this sits in an exact sequence

$$
0 \longrightarrow \underline{\operatorname{Hom}}\left(H, \mathbb{G}_{m}\right) \longrightarrow \operatorname{Pic}_{Y / S}^{0} \longrightarrow \operatorname{Pic}_{X / S}^{0} \longrightarrow 0 \text {. }
$$

Note that this can also be seen with the identification $\operatorname{Pic}_{X / S}^{0}=\underline{\operatorname{Ext}}^{1}\left(X, \mathbb{G}_{m}\right)$ explained in $[$ Gro72, Exposé VII] and the long exact Ext sequence.

Now suppose that the base scheme $S$ has characteristic $p>0$. Let $\mathscr{H} \subset \operatorname{Lie}_{X / S}$ be a subsheaf that is locally a direct summand and stable under the $p$-map, and let $H \subset X$ be the corresponding family of group schemes of height at most one. Then the quotient $Y=X / H$ is again a family of $g$-dimensional abelian varieties, and the projection $X \rightarrow Y$ induces an exact sequence (2.1). 


\section{RÖSSLER AND S. SCHRÖER}

Likewise, $0 \rightarrow H^{\prime} \rightarrow Y \rightarrow X^{(p)} \rightarrow 0$ with $H^{\prime}=X[F] / H$ gives a short exact sequence

$$
0 \longrightarrow \underline{\operatorname{Hom}}\left(H^{\prime}, \mathbb{G}_{m}\right) \longrightarrow \operatorname{Pic}_{X^{(p)} / S}^{0} \longrightarrow \operatorname{Pic}_{Y / S}^{0} \longrightarrow 0 \text {. }
$$

Write $\varphi: Y \rightarrow S$ and $\psi: X \rightarrow S$ for the structure morphisms.

Proposition 2.1. Suppose that the $p$-map $\operatorname{Lie}_{X / S} \rightarrow \operatorname{Lie}_{X / S}$ factors over the subsheaf $\mathscr{H}$. Then we have a four-term exact sequence

$$
0 \longrightarrow \mathscr{K} \longrightarrow R^{1} \psi_{*}\left(\mathscr{O}_{X}\right)^{(p)} \longrightarrow R^{1} \varphi_{*}\left(\mathscr{O}_{Y}\right) \longrightarrow \mathscr{K}^{(p)} \longrightarrow 0
$$

with the sheaf $\mathscr{K}=\mathscr{H} o m\left(\operatorname{Lie}_{X / S} / \mathscr{H}, \mathscr{O}_{S}\right)$. Moreover, the sequence is natural with respect to the inclusion $H \subset X$.

Proof. Consider the family $H^{\prime}=X[F] / H$ of group schemes of height at most one. Its Cartier dual $K=\underline{\operatorname{Hom}}\left(H^{\prime}, \mathbb{G}_{m}\right)$ is a family of finite group schemes. The latter have height at most one, by our assumption on the $p$-map on $\operatorname{Lie}_{X / S}$. We see from (1.2) that $H^{\prime}$ and $K$ are the Frobenius kernels for the respective vector $\operatorname{schemes} \operatorname{Spec}\left(\operatorname{Sym} \bullet\left(\mathscr{H}^{\prime \vee}\right)\right)$ and $\operatorname{Spec}\left(\operatorname{Sym}^{\bullet}\left(\mathscr{K}^{\vee}\right)\right)$, where $\mathscr{H}^{\prime}=\mathrm{Lie}_{H^{\prime} / S}$ and $\mathscr{K}=\mathrm{Lie}_{K / S}$.

By Lemma 2.2 below, the sheaf of Lie algebras $\mathscr{K}$ coincides with the linear dual of $\mathscr{H}^{\prime}=$ $\mathrm{Lie}_{X / S} / \mathscr{H}$. Our assertion is now a consequence of Theorem 1.2, applied to the short exact sequence (2.2). The four-term sequence is natural with respect to $H \subset X$ because the same holds for the two short exact sequences (2.1) and (2.2).

The preceding proof relies on the following observations: Let (LocLib/S) be the category of locally free sheaves of finite rank and $(\mathrm{Grp} / S)$ be the category of families of algebraic group schemes. Consider the functors

$$
\mathscr{E} \longmapsto V \text { and } \mathscr{E} \longmapsto V^{*}
$$

where $V=\operatorname{Spec}(\operatorname{Sym} \bullet(\mathscr{E} \vee))$ and $V^{*}=\operatorname{Spec}(\operatorname{Sym} \bullet(\mathscr{E}))$ are the vector bundles with $\operatorname{Lie}_{V / S}=\mathscr{E}$ and $\mathrm{Lie}_{V^{*} / S}=\mathscr{E} \mathrm{V}$, with trivial Lie brackets and $p$-maps. Note that we follow Grothendieck's convention from [GD60, Section 9.6]. The Frobenius kernels $G=V[F]$ and $G^{*}=V^{*}[F]$ are families of finite local group schemes.

LEMma 2.2. The contravariant functors $\mathscr{E} \mapsto G^{*}$ and $\mathscr{E} \mapsto \underline{\operatorname{Hom}}\left(G, \mathbb{G}_{m}\right)$ are naturally isomorphic. In particular, there is an identification

$$
\operatorname{Lie}_{\underline{\operatorname{Hom}}\left(G, \mathbb{G}_{m}\right) / S}=\mathscr{H} o m\left(\operatorname{Lie}_{G / S}, \mathscr{O}_{S}\right)
$$

of locally free sheaves that is natural in $G$.

Proof. The natural identification arises as follows: Let $T=\operatorname{Spec}(A)$ be an affine $S$-scheme, and consider the resulting $A$-module $E=\Gamma\left(T, \mathscr{E}_{T}\right)$, which is finitely generated and projective. According to (1.1), the group of $A$-valued points in the Cartier dual $\underline{\operatorname{Hom}}\left(G, \mathbb{G}_{m}\right)$ is the set of linear maps

$$
E=\mathrm{Lie}_{G / S} \otimes A \longrightarrow \mathrm{Lie}_{\mathbb{G}_{m} / S} \otimes A=A
$$

that are compatible with $p$-maps. On the left-hand side, the $p$-map vanishes, whereas on the right-hand side, it is nothing but $\lambda \mapsto \lambda^{p}$. So these linear maps can be seen as vectors in the dual $\operatorname{Hom}_{A}(E, A)$ annihilated by the relative Frobenius map. The latter coincide with the $A$-valued points of the Frobenius kernel $G^{*}=V^{*}[F]$.

The following property of families of abelian varieties $\varphi: Y \rightarrow S$ will be crucial for later computations. 
Proposition 2.3. For all $s \geqslant 0$, the higher direct image sheaves $R^{s} \varphi_{*}\left(\mathscr{O}_{Y}\right)$ are locally free, their formation commutes with base change, and the canonical maps $\Lambda^{s} R^{1} \varphi_{*}\left(\mathscr{O}_{Y}\right) \rightarrow R^{s} \varphi_{*}\left(\mathscr{O}_{Y}\right)$ are bijective. Moreover, the Leray-Serre spectral sequence

$$
E_{2}^{r, s}=H^{r}\left(S, R^{s} \varphi_{*}\left(\mathscr{O}_{Y}\right)\right) \Longrightarrow H^{r+s}\left(Y, \mathscr{O}_{Y}\right)
$$

has trivial differentials on the $E_{i}$-page provided that $p-1$ does not divide $i-1$.

Proof. The first assertion is [BBM82, Proposition 2.5.2]. For the second assertion, note that the differentials on the $i$ th page are certain additive maps $d_{i}: E_{i}^{r, s} \rightarrow E_{i}^{r+i, s-i+1}$, which are natural with respect to the family of abelian varieties $Y$. In particular, for each integer $n$, the multiplication on $Y$ induces an endomorphism $n^{*}$ on $\Lambda^{s} R^{1} \varphi_{*}\left(\mathscr{O}_{Y}\right)$. The latter is multiplication by $n^{s}$. To check this, it suffices to treat the case where $S=\operatorname{Spec}(R)$ is affine and $s=1$. Then the families of abelian varieties form an additive category, and $Y \mapsto H^{1}\left(Y, \mathscr{O}_{Y}\right)$ is a contravariant functor into the additive category of all $R$-modules, with the property $H^{1}\left(\mathscr{O}_{Y_{1} \times Y_{2}}\right)=H^{1}\left(\mathscr{O}_{Y_{1}}\right) \oplus H^{1}\left(\mathscr{O}_{Y_{2}}\right)$. Now recall that any functor $F: \mathcal{C} \rightarrow \mathcal{C}^{\prime}$ between additive categories that respects products also respects the $\mathbb{Z}$-module structure on hom sets [KS06, Proposition 8.2.15], and thus $n^{*}=n$.

Now suppose that there is an element $a \in E_{i}^{r, s}$ whose image $b \in E_{i}^{r+i, s-i+1}$ is non-zero. The latter can be seen as a basis vector inside an $\mathbb{F}_{p}$-vector space. Recall that the multiplicative group $\mathbb{F}_{p}^{\times}$is cyclic of order $p-1$. Choose an integer $n$ that generates $\mathbb{F}_{p}^{\times}$. Since $p-1 \nmid i-1$, we have $n^{i-1} \not \equiv 1$ modulo $p$. One gets

$$
n^{s} b=n^{s} d_{i}(a)=d_{i}\left(n^{s} a\right)=n^{s-i+1} d_{i}(a)=n^{s-i+1} b
$$

from the naturality of the Leray-Serre spectral sequence applied to $n^{*}$. Comparing coefficients gives $n^{1-i} \equiv 1$ modulo $p$ and therefore a contradiction.

\section{Moret-Bailly families}

Let $k$ be a ground field of characteristic $p>0$. Recall that an abelian variety $A$ of dimension $g \geqslant 1$ is called superspecial if the Lie algebra $\mathfrak{g}=\operatorname{Lie}(A)$ has trivial $p$-map. For $g=1$, this means that $A=E$ is a supersingular elliptic curve. Moreover, the products $A=E_{1} \times \cdots \times E_{g}$ of supersingular elliptic curves are superspecial. Note that if $k$ is algebraically closed, the converse holds [Oor75, Theorem 2]. If moreover $g \geqslant 2$, the isomorphism class of $A$ does not depend on the factors [Shi79, Theorem 3.5]. We need the following well-known existence result.

LEMma 3.1. In each dimension $g \geqslant 1$, there is a superspecial abelian variety $A$.

Proof. Using the three Weierstraß equations

$$
y^{2}+x y=x^{3}+\frac{36}{1728-j} x+\frac{1}{1728-j} \quad \text { and } \quad y^{2}+y=x^{3} \quad \text { and } \quad y^{2}=x^{3}+x,
$$

one sees that each invariant $j \in k$ is attained by some elliptic curve (compare with [Tat75, Equation (2.4) on p. 36]). So it suffices to show that there are supersingular $j$-values in the prime field $k=\mathbb{F}_{p}$. For $p=2$, this is $j=0$. Now suppose $p \geqslant 3$. Recall that an elliptic curve in Legendre form $E: y^{2}=x(x-1)(x-\lambda)$ is supersingular if and only if $\lambda$ is a root of the Hasse polynomial $P(T)=\sum_{i=0}^{m}\left(\begin{array}{c}m \\ i\end{array}\right)^{2} T^{i}$, where $m=(p-1) / 2$. One may view the spectrum of $k[\lambda]$ as the coarse moduli space for elliptic curves $E$ with level structure $(\mathbb{Z} / 2 \mathbb{Z})_{k}^{2} \subset E$. The group $G=\mathrm{GL}_{2}\left(\mathbb{F}_{2}\right)$ acts freely via the level structures, and the ring of invariants $k[\lambda]^{G}=k[j]$ is the coarse moduli space for the Deligne-Mumford stack $\mathscr{M}_{1,1}$. According to [BM04, Theorem 1], the Hasse polynomial has at least one root over $k$ if and only if $p \not \equiv 1$ modulo 4 ; then a supersingular 


\section{RÖSSLER AND S. SCHRÖER}

$E$ over $\mathbb{F}_{p}$ already exists with level structure and can be put in Legendre form. Now suppose $p \equiv 1$ modulo 4 , and consider the subgroup $H \subset G$ generated by interchanging the 2-division points with coordinate $x=0,1$. This is given by the change of coordinates $x=-x^{\prime}+1$ and induces $\lambda \mapsto 1-\lambda$ on the coarse moduli space. By loc. cit., the Hasse polynomial viewed as element in the ring of invariants $k[\lambda]^{H}=k\left[\lambda-\lambda^{2}\right]$ acquires a root, which gives the desired supersingular $j$-value.

Fix some integers $n, d \geqslant 1$, and choose a superspecial abelian variety $A$ of dimension $g=n+1$. In turn, every non-zero vector in $\mathfrak{g}=\operatorname{Lie}(A)$ gives an inclusion $\alpha_{p} \subset A$. Choose an identification $\mathfrak{g}=k^{n+1}$. Set $X=A \times \mathbb{P}^{n}$, and view this as the constant family of abelian varieties over $\mathbb{P}^{n}=\operatorname{Proj} k\left[T_{0}, \ldots, T_{n}\right]=\mathbb{P}\left(\mathfrak{g}^{\vee}\right)$. Choose some homogeneous polynomials $Q_{0}, \ldots, Q_{n}$ of degree $d \geqslant 1$ without common zero on the projective space, and consider the resulting inclusion

$$
\mathscr{O}_{S}(-d) \subset \mathscr{O}_{\mathbb{P}^{n}}^{\oplus n+1}=\mathfrak{g} \otimes_{k} \mathscr{O}_{\mathbb{P}^{n}}=\operatorname{Lie}_{X / \mathbb{P}^{n}}
$$

Let $H \subset X$ be the family of height one group schemes with $\operatorname{Lie}_{H / S}=\mathscr{O}_{S}(-d)$, and form the resulting quotient $Y=X / H$. This is smooth and proper, of $\operatorname{dimension} \operatorname{dim}(Y)=2 n+1$, and with $h^{0}\left(\mathscr{O}_{Y}\right)=1$. The inclusion $H \subset A[p] \times \mathbb{P}^{n}$ induces a finite morphism $Y \rightarrow(A / A[F]) \times \mathbb{P}^{n}$; hence $Y$ is projective. Write

$$
\varphi: Y=X / H=\left(A \times \mathbb{P}^{n}\right) / H \longrightarrow \mathbb{P}^{n}
$$

for the structure morphism, which is a family of supersingular abelian varieties of dimension $g=n+1$, and $\epsilon: X \rightarrow Y$ for the quotient map.

We call $Y$ a Moret-Bailly family because the above generalizes the pencils in [Mor81, Part 2] (where the case $n=1$ and $d=1$ is considered) to arbitrary dimensions. Note that the construction $Y=Y_{A, q}$ depends on the superspecial abelian variety $A$ and the finite flat morphism $q: \mathbb{P}^{n} \rightarrow \mathbb{P}^{n}$ defined by the homogeneous polynomials $Q_{i}$, but we usually neglect this in notation.

The cokernel $\mathscr{E}_{d}$ for the inclusion $\mathscr{O}_{\mathbb{P}^{n}}(-d) \subset \mathfrak{g} \otimes \mathscr{O}_{\mathbb{P}}$ is locally free and sits in the short exact sequence

$$
0 \longrightarrow \mathscr{O}_{\mathbb{P}^{n}}(-d) \longrightarrow \mathscr{O}_{\mathbb{P}^{n}}^{\oplus n+1} \longrightarrow \mathscr{E}_{d} \longrightarrow 0
$$

thus has $\operatorname{det}\left(\mathscr{E}_{d}\right)=\mathscr{O}_{\mathbb{P}^{n}}(d)$. Note that for $Q_{i}=T_{i}$, this becomes the Euler sequence (compare with [OSS80, Section 1.1, p. 6]), and $\mathscr{E}_{1}=\Theta_{\mathbb{P}^{n} / k}(-1)$. In general, we have $\mathscr{E}_{d}=q^{*}\left(\Theta_{\mathbb{P}^{n} / k}(-1)\right)$, where $q: \mathbb{P}^{n} \rightarrow \mathbb{P}^{n}$ is the morphism of $\operatorname{degree} \operatorname{deg}(q)=d^{n}$ defined by the homogeneous polynomials $Q_{i}$.

Note that the Frobenius pullback $\mathscr{E}_{p d}=\mathscr{E}_{d}^{(p)}$ is obtained by taking the p-powers $Q_{0}^{p}, \ldots, Q_{n}^{p}$. To simplify notation, we write $\mathscr{E}_{-d}=\underline{\operatorname{Hom}}\left(\mathscr{E}_{d}, \mathscr{O}_{\mathbb{P}}\right)$ for the dual sheaves and also set $\mathscr{E}_{-p d}=\mathscr{E}_{-d}^{(p)}$.

Proposition 3.2. The sheaf of Lie algebras and the first direct image are given by

$$
\operatorname{Lie}_{Y / \mathbb{P}^{n}}=\mathscr{E}_{d} \oplus \mathscr{O}_{\mathbb{P}^{n}}(-d p) \quad \text { and } \quad R^{1} \varphi_{*}\left(\mathscr{O}_{Y}\right)=\mathscr{O}_{\mathbb{P}^{n}}(d) \oplus \mathscr{E}_{-p d}
$$

Moreover, the p-map in $\operatorname{Lie}_{Y / \mathbb{P}^{n}}$ is trivial on the first summand and sends the second summand to the first for all such splittings.

Proof. By Theorem 1.2, the sheaf of Lie algebras is an extension of $\mathscr{O}_{\mathbb{P}^{n}}(-p d)$ by $\mathscr{E}_{d}$. All such extensions split: We have $\operatorname{Ext}^{1}\left(\mathscr{O}_{\mathbb{P}^{n}}(-p d), \mathscr{E}_{d}\right)=H^{1}\left(\mathbb{P}^{n}, \mathscr{E}_{d}(p d)\right)$, and tensoring (3.1) with $\mathscr{O}_{\mathbb{P}^{n}}(p d)$ yields an exact sequence

$$
H^{1}\left(\mathbb{P}^{n}, \mathfrak{g} \otimes_{k} \mathscr{O}_{\mathbb{P}^{n}}(p d)\right) \longrightarrow H^{1}\left(\mathbb{P}^{n}, \mathscr{E}_{d}(p d)\right) \longrightarrow H^{2}\left(\mathbb{P}^{n}, \mathscr{O}_{\mathbb{P}}(p d-d)\right)
$$

The outer terms vanish because the invertible sheaves $\mathscr{O}_{\mathbb{P} n}(p d)$ and $\mathscr{O}_{\mathbb{P}}(p d-d)$ are ample. Thus $\operatorname{Lie}_{Y / \mathbb{P} n}$ splits. 


\section{Moret-BAilly fAMilies AND NON-LIFTABle SCHEMES}

Next we verify the assertion on the $p$-map. By construction, $\mathscr{E}_{d}$ is a quotient for $\mathrm{Lie}_{X / S}$, whereas $\mathscr{O}_{\mathbb{P}^{n}}(-p d)$ is a subsheaf of $\operatorname{Lie}_{X^{(p)} / S}$. Since $X=A \times \mathbb{P}^{n}$ comes from a superspecial abelian variety $A$, the $p$-maps vanish on these sheaves of Lie algebras, and the assertion follows.

It remains to analyze the first direct image. The coherent sheaf

$$
\mathscr{K}=\underline{\operatorname{Hom}}\left(\mathscr{O}_{X}^{\oplus n+1} / \mathscr{O}_{\mathbb{P}^{n}}(-d), \mathscr{O}_{\mathbb{P}^{n}}\right)=\underline{\operatorname{Hom}}\left(\mathscr{E}_{d}, \mathscr{O}_{\mathbb{P}^{n}}\right)
$$

is nothing but $\mathscr{E}_{-d}$, and its Frobenius pullback becomes $\mathscr{K}^{(p)}=\mathscr{E}_{-p d}$. According to Proposition 2.1, we have a four-term exact sequence

$$
0 \longrightarrow \mathscr{E}_{-d} \longrightarrow \mathscr{O}_{\mathbb{P}^{n}}^{\oplus n+1} \longrightarrow R^{1} \varphi_{*}\left(\mathscr{O}_{Y}\right) \longrightarrow \mathscr{E}_{-p d} \longrightarrow 0 .
$$

The inclusion on the left is locally a direct summand, so its cokernel is isomorphic to $\mathscr{O}_{\mathbb{P} n}(d)$, which follows by taking determinants. Thus the direct image $R^{1} \varphi_{*}\left(\mathscr{O}_{Y}\right)$ is an extension of $\mathscr{E}_{-p d}$ by $\mathscr{O}_{\mathbb{P}^{n}}(d)$. As in the preceding paragraph, one verifies that all such extensions split.

This computation has the following consequence.

Corollary 3.3. For each rational point $a \in \mathbb{P}^{n}$, there are only finitely many other rational points $b \in \mathbb{P}^{n}$ such that $\varphi^{-1}(a) \simeq \varphi^{-1}(b)$. Moreover, any field of definition $F$ for the generic fiber $\varphi^{-1}(\eta)$ has $\operatorname{trdeg}(F)=n$.

Proof. We may assume that $k$ is algebraically closed. Choose some odd prime $l \neq p$ and some symplectic level structure $(\mathbb{Z} / l \mathbb{Z})^{2 g} \rightarrow A$. This descents to a family of symplectic level structures for $Y$. Let $\mathscr{A}_{g, l}$ be the Artin stack of $g$-dimensional abelian varieties endowed with such a structure. This is actually an algebraic space [FC90, Corollary 2 for Theorem 6.7] that is separated and of finite type. Our Moret-Bailly family corresponds to a morphism $h: \mathbb{P}^{n} \rightarrow \mathscr{A}_{g, l}$. It suffices to check that $h$ is quasi-finite because every abelian variety has only finitely many such level structures. Suppose that it is not quasi-finite. Then there is an integral curve $C \subset \mathbb{P}^{n}$ that maps to a closed point. Then the restriction $Y_{C}=Y \times_{\mathbb{P}^{n}} C$ is isomorphic to $B_{C}=B \times C$ for some abelian variety $B$. Passing to the sheaf of Lie algebras, we get $\left(\mathscr{E}_{d} \oplus \mathscr{O}_{\mathbb{P}} n(-d p)\right)_{C} \simeq \mathscr{O}_{C}^{\oplus n+1}$ as coherent sheaves, in contradiction to the Krull-Schmidt theorem for coherent sheaves [Ati56, Theorem 1]. The assertion on the field of definition is proven in an analogous way.

To simplify the notation, we now write $\mathscr{O}_{Y}(m)$ for the preimages under the structure morphism $\varphi: Y \rightarrow \mathbb{P}^{n}$ of the invertible sheaves $\mathscr{O}_{\mathbb{P}^{n}}(m)$.

Corollary 3.4. The dualizing sheaf takes the form $\omega_{Y}=\mathscr{O}_{Y}(m)$ for the integer $m=d(p-1)-$ $(n+1)$. In particular, $c_{1}=0$ holds if and only if $d(p-1)=n+1$, and in this case we actually have $\omega_{Y}=\mathscr{O}_{Y}$.

Proof. Since $Y$ is smooth, the dualizing sheaf is $\omega_{Y}=\operatorname{det}\left(\Omega_{Y / k}^{1}\right)$. We have a short exact sequence $0 \rightarrow \varphi^{*}\left(\Omega_{\mathbb{P}^{n} / k}^{1}\right) \rightarrow \Omega_{Y / k}^{1} \rightarrow \Omega_{Y / \mathbb{P}^{n}}^{1} \rightarrow 0$. The cokernel is isomorphic to the preimage of the dual for $\operatorname{Lie}_{Y / \mathbb{P}^{n}}$ because $\varphi: Y \rightarrow \mathbb{P}^{n}$ is a family of smooth algebraic group schemes. The sheaf of Lie algebras equals $\mathscr{E}_{d} \oplus \mathscr{O}_{\mathbb{P}^{n}}(-p d)$, and we have $\operatorname{det}\left(\mathscr{E}_{d}\right)=\mathscr{O}_{\mathbb{P}^{n}}(d)$. Furthermore, $\operatorname{det}\left(\Omega_{\mathbb{P}^{n}}^{1}\right)=$ $\mathscr{O}_{\mathbb{P}^{n}}(-n-1)$. Combining all this, we obtain $\omega_{Y}=\mathscr{O}_{Y}(m)$ for the integer $m=-n-1-d+p d$.

It follows that the Kodaira dimension takes the values $\operatorname{Kod}(Y) \in\{-\infty, 0, n\}$ depending on the sign of the integer $m=d(p-1)-(n+1)$. Moreover, in the canonical model, $Z=$ Proj $\bigoplus_{t \geqslant 0} H^{0}\left(Y, \omega^{\otimes t}\right)$ is given by the respective schemes $Z=\varnothing, \mathbb{P}^{0}, \mathbb{P}^{n}$. Analogous statements hold for the anticanonical models. 


\section{RÖSSLER AND S. SCHRÖER}

It is easy to determine the Betti numbers $b_{i} \geqslant 0$, defined as the ranks of the $l$-adic cohomology groups $H^{i}\left(\bar{Y}, \mathbb{Z}_{l}(i)\right)=\lim _{\nu \geqslant 0} H^{i}\left(\bar{Y}, \mu_{l^{\nu}}^{\otimes i}\right)$, where $\bar{Y}=Y \otimes k^{\text {alg }}$ is the base change to some algebraic closure and $l>0$ is a prime different from $p$.

Proposition 3.5. The l-adic cohomology groups $H^{i}\left(\bar{Y}, \mathbb{Z}_{l}(i)\right)$ are free of rank $b_{i}=\sum_{j}\left(\begin{array}{c}2 n+2 \\ i-j\end{array}\right)$, where the sum runs over all even $j \geqslant 0$. In particular, we have $b_{1}=2 n+2$ and $b_{2}=2 n^{2}+3 n+2$ and $b_{2 n+1}=2^{2 n+1}$.

Proof. We may assume that $k$ is algebraically closed. The quotient map $\epsilon: X \rightarrow Y$ is a finite universal homeomorphism, so the $l$-adic cohomology groups for $Y=X / H$ and $X=A \times \mathbb{P}^{n}$ coincide. Taking cohomology with coefficients in $R=\mathbb{Z} / l^{\nu} \mathbb{Z}$, we have $H^{\bullet}\left(\mathbb{P}^{n}\right)=R[h] /\left(h^{n+1}\right)$ and $H^{\bullet}(A)=\Lambda^{\bullet} H^{1}(A)$. These $R$-modules are free, where the generator $h$ has degree two and $H^{1}(A)$ is of rank $2 n+2$. In turn,

$$
H^{i}(X)=\bigoplus_{j} H^{j}\left(\mathbb{P}^{n}\right) \otimes_{R} H^{i-j}(A)
$$

by the Künneth formula [AGV73, Exposé XVII, Theorem 5.4.3], and the assertion on $H^{i}\left(Y, \mathbb{Z}_{l}(i)\right)$ is a direct consequence. The values $b_{1}$ and $b_{2}$ follow immediately. In middle degree, we get $b_{2 n+1}=$ $\sum_{s}\left(\begin{array}{c}2 n+2 \\ s\end{array}\right)$, where the sum runs over all odd $s$. This sum is half of $(1+1)^{2 n+2}-(1-1)^{2 n+2}=$
$2^{2 n+2}$.

Note that since $b_{2 n+1} \neq 0$, the method introduced by Hirokado [Hir99] to establish nonliftability apparently does not apply.

\section{The Picard scheme and the Albanese map}

Keep the notation from the previous section, so that $Y=\left(A \times \mathbb{P}^{n}\right) / H$ is a Moret-Bailly family formed with some superspecial abelian variety $A$ of dimension $g=n+1$ and some homogeneous polynomials $Q_{0}, \ldots, Q_{n}$ of degree $d \geqslant 1$ without common zero on $\mathbb{P}^{n}$. Let $\varphi: Y \rightarrow \mathbb{P}^{n}$ be the structure morphism. We now examine the Picard scheme for $Y$. Recall that its Lie algebra is the cohomology group $H^{1}\left(Y, \mathscr{O}_{Y}\right)$.

Proposition 4.1. The Picard scheme $\operatorname{Pic}_{Y / k}$ has dimension $n+1$, and furthermore $h^{1}\left(\mathscr{O}_{Y}\right)=$ $\left(\begin{array}{c}n+d \\ d\end{array}\right)$. In particular, the Picard scheme is smooth if and only if $d=1$.

Proof. We may assume that $k$ is algebraically closed. To compute the dimension $d \geqslant 0$ of the Picard scheme, choose a prime $l \neq p$ that does not divide the order of the torsion part in $\mathrm{NS}(Y)$. The Kummer sequence $0 \rightarrow \mu_{l} \rightarrow \mathbb{G}_{m} \rightarrow \mathbb{G}_{m} \rightarrow 0$ implies that $2 d=b_{1}$. According to Proposition 3.5, we have $b_{1}=2 n+2$.

The Leray-Serre spectral sequence for $\varphi: Y \rightarrow \mathbb{P}^{n}$ gives an exact sequence

$$
0 \longrightarrow H^{1}\left(\mathbb{P}^{n}, \mathscr{O}_{\mathbb{P}^{n}}\right) \rightarrow H^{1}\left(Y, \mathscr{O}_{Y}\right) \longrightarrow H^{0}\left(\mathbb{P}^{n}, R^{1} \varphi_{*}\left(\mathscr{O}_{Y}\right)\right) \longrightarrow H^{2}\left(\mathbb{P}^{n}, \mathscr{O}_{\mathbb{P}^{n}}\right)
$$

The outer terms vanish, and we merely have to compute the global sections of $R^{1} \varphi_{*}\left(\mathscr{O}_{Y}\right)=$ $\mathscr{O}_{\mathbb{P}}(d) \oplus \mathscr{E}_{-p d}$. The first summand contributes $h^{0}\left(\mathscr{O}_{\mathbb{P}}(d)\right)=\left(\begin{array}{c}n+d \\ d\end{array}\right)$. It remains to check that the sheaf $\mathscr{E}_{-p d}$ has no non-zero global sections. Dualizing the short exact sequence $0 \rightarrow \mathscr{O}_{\mathbb{P}^{n}}(-p d) \rightarrow$ $\mathscr{O}_{\mathbb{P}^{n}}^{\oplus n+1} \rightarrow \mathscr{E}_{p d} \rightarrow 0$, we get an exact sequence

$$
0 \longrightarrow H^{0}\left(\mathbb{P}^{n}, \mathscr{E}_{-p d}\right) \longrightarrow H^{0}\left(\mathbb{P}^{n}, \mathscr{O}_{\mathbb{P}^{n}}^{\oplus n+1}\right) \longrightarrow H^{0}\left(\mathbb{P}^{n}, \mathscr{O}_{\mathbb{P}^{n}}(p d)\right)
$$




\section{Moret-BAilly fAMilies AND NON-LIFTABLE SCHEMES}

The map on the right is given by the homogeneous polynomials $Q_{0}^{p}, \ldots, Q_{n}^{p}$. These are linearly independent, so the map must be injective. It follows that $H^{0}\left(\mathbb{P}^{n}, \mathscr{E}_{-p d}\right)=0$. This shows $h^{1}\left(\mathscr{O}_{Y}\right)=\left(\begin{array}{c}n+d \\ d\end{array}\right)$.

Set $X=A \times \mathbb{P}^{n}$. The family $H \subset X$ of finite group schemes of height one sits inside the constant family $X[F]=A[F] \times \mathbb{P}^{n}$. In turn, we get an induced homomorphism $Y \rightarrow A^{(p)} \times$ $\mathbb{P}^{n}$ between families of abelian varieties and write $\psi: Y \rightarrow A^{(p)}$ for the composition with the projection.

Proposition 4.2. The morphism $\psi: Y \rightarrow A^{(p)}$ is flat, and every geometric fiber is non-reduced, with reduction isomorphic to the projective $n$-space. Moreover, the canonical map $\mathscr{O}_{A^{(p)}} \rightarrow$ $\psi_{*}\left(\mathscr{O}_{Y}\right)$ is bijective.

Proof. The quotient map $\epsilon: X \rightarrow Y$ is faithfully flat, and so is the composition $\psi \circ \epsilon: X=$ $A \times \mathbb{P}^{n} \rightarrow A^{(p)}$. By descent, $\psi: Y \rightarrow A^{(p)}$ must be flat.

The fiber $Z=\psi^{-1}(0)$ over the origin is the family $\left(A[F] \times \mathbb{P}^{n}\right) / H$ of height one group schemes, with $\operatorname{Lie}_{Z / \mathbb{P}^{n}}=\mathscr{E}_{d}$. We claim that $h^{0}\left(\mathscr{O}_{Z}\right)=1$. The universal enveloping algebra is $U\left(\mathscr{E}_{d}\right)=\operatorname{Sym}^{\bullet}\left(\mathscr{E}_{d}\right)$, and the restricted quotient becomes $U^{[p]}\left(\mathscr{E}_{d}\right)=\operatorname{Sym}^{\bullet}\left(\mathscr{E}_{d}\right) / \mathscr{E}_{d}^{(p)} \operatorname{Sym}^{\bullet}\left(\mathscr{E}_{d}\right)$. According to (1.2), the fiber $Z$ is the relative spectrum of the corresponding sheaf of Hopf algebras

$$
\mathscr{A}=\mathscr{H} o m\left(U^{[p]}\left(\mathscr{E}_{d}\right), \mathscr{O}_{\mathbb{P} n}\right) \subset \mathscr{H} o m\left(\operatorname{Sym} \bullet\left(\mathscr{E}_{d}\right), \mathscr{O}_{\mathbb{P}^{n}}\right)
$$

The term on the right is the product of the coherent sheaves $\mathscr{H} o m\left(\operatorname{Sym}^{i}\left(\mathscr{E}_{d}\right), \mathscr{O}_{\mathbb{P}^{n}}\right)$, and the term on the left is already contained in the corresponding sum. The summands are divided powers $\Gamma^{i}(\mathscr{F})=\operatorname{Sym}^{i}\left(\mathscr{F}^{\vee}\right)^{\vee}$ for the dual sheaf $\mathscr{F}=\mathscr{E}_{d}^{\vee}$. It thus suffices to verify that the divided powers have no non-zero global sections for $i \geqslant 1$. We proceed by induction. The case $i=1$ was already treated in the proof for Proposition 4.1. Now suppose $i \geqslant 2$ and that the assertion is true for $i-1$. The surjection $\mathscr{O}_{\mathbb{P} n}^{\oplus n+1} \rightarrow \mathscr{E}_{d}$ induces a canonical surjection $\operatorname{Sym}^{i-1}\left(\mathscr{E}_{d}\right) \otimes \mathscr{O}_{\mathbb{P} n}^{\oplus n+1} \rightarrow \operatorname{Sym}^{i}\left(\mathscr{E}_{d}\right)$. Dualizing the latter gives an inclusion $\Gamma^{i}(\mathscr{F}) \subset \Gamma^{i-1}(\mathscr{F}) \otimes \mathscr{O}_{\mathbb{P} n}^{\oplus n+1}$, which completes the induction. Note that the inclusion is a piece from the Eagon-Northcott complex [EN62]. Summing up, this establishes $h^{0}\left(\mathscr{O}_{Z}\right)=1$.

Now consider the fiber over a geometric point $\bar{b}$ : $\operatorname{Spec}(\Omega) \rightarrow A^{(p)}$, with image $b \in A^{(p)}$. Making a base change, it suffices to treat the case where $k=\Omega$ is algebraically closed and $b \in A^{(p)}$ is rational. Then $b=a^{(p)}$ for some rational point $a \in A$, and $\psi^{-1}(b)=\left((a+A[F]) \times \mathbb{P}^{n}\right) / H$. This is isomorphic to $\psi^{-1}(0)=\left(A[F] \times \mathbb{P}^{n}\right) / H$ via translation by $a$, so the fiber is non-reduced, with reduction isomorphic to $\mathbb{P}^{n}$.

It remains to compute $\psi_{*}\left(\mathscr{O}_{Y}\right)$. We just saw that the function $b \mapsto h^{0}\left(\mathscr{O}_{Y_{b}}\right)=1$ is constant on the reduced scheme $A^{(p)}$. It follows that the direct image sheaf is locally free of rank one, hence the canonical map $\mathscr{O}_{A^{(p)}} \rightarrow \psi_{*}\left(\mathscr{O}_{Y}\right)$ is bijective.

As explained by Serre [Ser60], there is a morphism $Y \rightarrow V$ to some abelian variety $V$ such that every other such morphism $Y \rightarrow V^{\prime}$ arises via composition with some unique $V \rightarrow V^{\prime}$. Note that the latter usually does not respect the origin. This $V=\mathrm{Alb}_{Y / k}$ is called the Albanese variety, and $Y \rightarrow \mathrm{Alb}_{Y / k}$ is the Albanese map. See [LS21] for a relative theory.

Proposition 4.3. The morphism $\psi: Y \rightarrow A^{(p)}$ is the Albanese map.

Proof. Let $f: Y \rightarrow B$ be a morphism into another abelian variety. We have to show that this map factors uniquely over $\psi: Y \rightarrow A^{(p)}$. The composition $f \circ \epsilon: X \rightarrow B$ factors over the projection 


\section{RÖSSLER AND S. SCHRÖER}

$\operatorname{pr}_{1}: X=A \times \mathbb{P}^{n} \rightarrow A$. This gives a commutative diagram

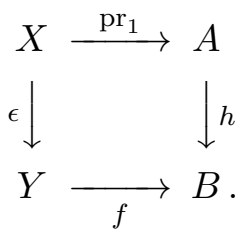

Replacing $f$ with the composition of a translation of $B$ with $f$, we may assume that $h: A \rightarrow B$ respects origins, hence is a homomorphism. For each rational point $a \in \mathbb{P}^{n}$, the fiber $H_{a} \subset X_{a}=A$ is a copy of $\alpha_{p}$ whose schematic image in $Y$ and hence also in $B$ is a rational point $b \in B$. The inclusion $\mathscr{O}_{\mathbb{P} n}(-d) \subset \mathscr{O}_{\mathbb{P} n}^{\oplus n+1}$ defining the family of subgroup schemes $H \subset X$ is locally a direct summand; hence the canonical map $\bigcup_{a} H_{a} \rightarrow A$ from the disjoint union has schematic image $A[F]$. In the commutative diagram

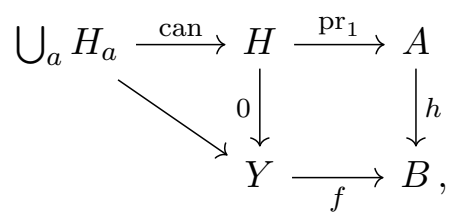

the composition $h \circ \mathrm{pr}_{1} \circ$ can factors over the origin $0 \in B$, and the schematic image of $\operatorname{pr}_{1} \circ$ can is $A[F]$. Thus $h: A \rightarrow B$ factors over $A / A[F]=A^{(p)}$. The factorization is unique because the composition $X \rightarrow A \rightarrow A / A[F]$ is faithfully flat, hence an epimorphism.

\section{Non-existence of projective liftings}

We keep the assumptions of the preceding section and furthermore assume $n \geqslant 2$ and $d(p-1) \leqslant$ $n+1$. These assumptions on $n, d, p$ are made only in this section. In this situation, our MoretBailly family $Y=\left(A \times \mathbb{P}^{n}\right) / H$ has dimension $\operatorname{dim}(Y)=2 n+1 \geqslant 5$ and $\omega_{Y}$ is anti-nef. Recall that one says that $Y$ projectively lifts to characteristic zero if there is a local noetherian $\operatorname{ring} R$ with residue field $k=R / \mathfrak{m}_{R}$ such that the canonical map $\mathbb{Z} \rightarrow R$ is injective, together with a projective flat morphism $\mathfrak{Y} \rightarrow \operatorname{Spec}(R)$ whose closed fiber is isomorphic to $Y$. Note that one may assume that $R$ is also complete and one-dimensional. The goal of this section is to establish the following.

TheOREm 5.1. The scheme $Y$ does not projectively lift to characteristic zero.

The proof requires some preparation and is given at the end of this section. It suffices to treat the case where $k$ is algebraically closed. Seeking a contradiction, we assume that $Y$ projectively lifts. Then there is a complete discrete valuation ring $R$ with residue field $R / \mathfrak{m}_{R}=k$ whose field of fractions $F=\operatorname{Frac}(R)$ has characteristic zero, together with a proper flat morphism $v: \mathfrak{Y} \rightarrow \operatorname{Spec}(R)$ with closed fiber $Y=\mathfrak{Y} \otimes_{R} k$. Write $V=\mathfrak{Y} \otimes_{R} F$ for the generic fiber, which is a smooth proper scheme with $h^{0}\left(\mathscr{O}_{V}\right)=1$.

We start by examining the Picard scheme of $V$. The component of the origin $P=\mathrm{Pic}_{V / F}^{0}$ is an abelian variety. Consider the dual abelian variety $\mathrm{Pic}_{P / F}^{0}$. After passing to a finite extension of $R$, we may assume that the structure morphism $\mathfrak{Y} \rightarrow \operatorname{Spec}(R)$ admits a section. In particular, the generic fiber $V$ contains a rational point. Then there is a Poincaré sheaf $\mathscr{P}$ on $V \times P$, and we may assume that it is numerically trivial on the fibers of the first projection. As explained in 


\section{Moret-BAilly fAMilies AND NON-LIFTABLE SCHEMES}

[Gro62, Theorem 3.3], the resulting

$$
\Psi: V \longrightarrow \operatorname{Pic}_{P / F}^{0}, \quad v \longmapsto[\mathscr{P} \mid\{v\} \times P]
$$

is the Albanese map, and we write $\mathrm{Alb}_{V / F}=\operatorname{Pic}_{P / F}^{0}$.

According to Proposition 4.3, the composition of the quotient map with the projection $A \times$ $\mathbb{P}^{n} \rightarrow A^{(p)}$ induces the Albanese map $\psi: Y \rightarrow A^{(p)}$, and the reduced preimage of the origin $Z=\psi^{-1}(0)_{\text {red }}$ is a copy of the projective $n$-space $\mathbb{P}_{k}^{n}$. We now exploit the existence of the relative Hilbert scheme Hilb $_{\mathfrak{Y} / R}$, which parameterizes flat families of closed subschemes [Gro61], and regard the closed subscheme $Z \subset Y$ as a $k$-valued point $\xi=[Z]$ in the relative Hilbert scheme.

Proposition 5.2. The structure morphism $\operatorname{Hilb}_{\mathfrak{Y} / R} \rightarrow \operatorname{Spec}(R)$ is smooth near $\xi$.

Proof. Let $\mathscr{I} \subset \mathscr{O}_{Y}$ be the sheaf of ideals for the closed subscheme $Z \subset Y$. According to [Gro61, Corollary 5.4], it suffices to check that the obstruction group $\operatorname{Ext}^{1}\left(\mathscr{I} / \mathscr{I}^{2}, \mathscr{O}_{Z}\right)$ vanishes. Since the inclusion $Z \subset Y$ is a regular embedding, the conormal sheaf $\mathscr{N}=\mathscr{I} / \mathscr{I}^{2}$ is locally free, and the obstruction group becomes $H^{1}\left(Z, \mathscr{N}^{\vee}\right)$.

To compute the sheaf $\mathscr{N}$, we consider the commutative diagram

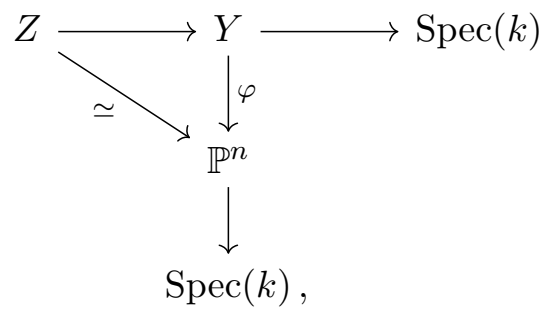

where the arrows are either smooth or regular embeddings. The vertical part yields a short exact sequence $0 \rightarrow \varphi^{*} \Omega_{\mathbb{P}^{n} / k}^{1} \rightarrow \Omega_{Y / k}^{1} \rightarrow \Omega_{Y / \mathbb{P}^{n}}^{1} \rightarrow 0$. The kernel is locally a direct summand, so the restriction

$$
0 \longrightarrow \varphi^{*} \Omega_{\mathbb{P}^{n} / k}^{1}\left|Z \longrightarrow \Omega_{Y / k}^{1}\right| Z \longrightarrow \Omega_{Y / \mathbb{P}^{n}}^{1} \mid Z \longrightarrow 0
$$

remains exact. The horizontal part yields another short exact sequence

$$
0 \longrightarrow \mathscr{I} / \mathscr{I}^{2} \longrightarrow \Omega_{Y / k}^{1} \mid Z \longrightarrow \Omega_{Z / k}^{1} \longrightarrow 0 \text {. }
$$

Using the commutativity of (5.1), we see that the inclusion of $\varphi^{*} \Omega_{\mathbb{P}^{n} / k}^{1} \mid Z$ splits the above extension, so the projection $\mathscr{I} / \mathscr{I}^{2} \rightarrow \Omega_{Y / \mathbb{P}^{n}}^{1} \mid Z$ is bijective. With the identification $Z=\mathbb{P}^{n}$ and Proposition 3.2, we conclude $\mathscr{N}^{\vee}=\mathscr{E}_{d} \oplus \mathscr{O}_{\mathbb{P}^{n}}(-p d)$.

One now easily checks that $H^{1}\left(Z, \mathscr{N}^{\vee}\right)=0$. Indeed, the short exact sequence $0 \rightarrow \mathscr{O}_{\mathbb{P}^{n}}(-d)$ $\rightarrow \mathscr{O}_{\mathbb{P}^{n}}^{\oplus n+1} \rightarrow \mathscr{E}_{d} \rightarrow 0$ yields an exact sequence

$$
H^{1}\left(\mathbb{P}^{n}, \mathscr{O}_{\mathbb{P}^{n}}^{\oplus n+1}\right) \longrightarrow H^{1}\left(\mathbb{P}^{n}, \mathscr{E}_{d}\right) \longrightarrow H^{2}\left(\mathbb{P}^{n}, \mathscr{O}_{\mathbb{P}^{n}}(-d)\right)
$$

The term on the left vanishes. This also holds for the term on the right for $n \neq 2$. Since $n=d(p-1) \geqslant d$, this also remains true for $n=2$. Furthermore, we have $H^{1}\left(\mathbb{P}^{n}, \mathscr{O}_{\mathbb{P} n}(-p d)\right)=0$ because $n \geqslant 2$.

By Hensel's lemma, the relative Hilbert scheme admits an $R$-valued point passing through $\xi \in$ Hilb $_{\mathfrak{Y} / R}$. Let $\mathfrak{Z} \subset \mathfrak{Y}$ be the corresponding flat family of closed subschemes, with closed fiber $\mathfrak{Z} \otimes_{R} k=Z=\mathbb{P}_{k}^{n}$. 


\section{RÖSSLER AND S. SCHRÖER}

Proposition 5.3. The generic fiber $\mathfrak{Z} \otimes_{R} F \subset \mathfrak{Y} \otimes_{R} F=V$ is isomorphic to $\mathbb{P}_{F}^{n}$ and must be contained in some fiber of the Albanese map $\Psi: V \rightarrow \mathrm{Alb}_{V / F}$.

Proof. Using $H^{1}\left(\mathbb{P}^{n}, \Theta_{\mathbb{P} n}\right)=0$, we inductively construct compatible isomorphisms $\mathbb{P}^{n} \otimes R / \mathfrak{m}_{R}^{n+1}$ $\rightarrow \mathfrak{Z} \otimes_{R} R / \mathfrak{m}_{R}^{n+1}$. Grothendieck's existence theorem gives $\mathbb{P}_{R}^{n} \simeq \mathfrak{Z}$. Since every morphism from the projective line to an abelian variety is constant, the scheme $\mathfrak{Z}_{F}$ must be contained in some fiber of $\Psi: V \rightarrow \operatorname{Alb}_{V / F}$.

Proof of Theorem 5.1. First note that by applying the specialization theorem [AGV73, Exposé XVI, Corollary 2.2] to the smooth proper morphism $\mathfrak{Y} \rightarrow \operatorname{Spec}(R)$, we may conclude that the Betti numbers of the closed and generic fibers coincide. In combination with Proposition 3.5, this shows that $b_{1}(V)=2(n+1)$. In turn, the abelian variety $\operatorname{Pic}_{V / F}^{0}$ has dimension $n+1$, and the same holds for the Albanese variety $\mathrm{Alb}_{V / F}$. Applying [Mor92, Corollary 8] to the dual of the relative dualizing sheaf of $\mathfrak{Y}$, we also see that the dualizing sheaf $\omega_{V}$ of $V$ is nef. The main result of [Cao19] now implies that the Albanese map $\Psi: V \rightarrow \mathrm{Alb}_{V / F}$ is smooth. In particular, the fibers of $\Psi$ are equidimensional of dimension $n=2 n+1-(n+1)$. By Proposition 5.3, there is a closed point $\lambda \in \mathrm{Alb}_{V / F}$ such that the fiber $V_{\lambda}=\Psi^{-1}(\lambda)$ of the Albanese map $\Psi$ contains the generic fiber $\mathfrak{Z} \otimes_{R} F$ of the family of subschemes $\mathfrak{Z} \subset \mathfrak{Y}$. Furthermore, by the same proposition, we have $\mathfrak{Z} \otimes_{R} F \simeq \mathbb{P}_{F}^{n}$. The inclusion $\mathfrak{Z} \otimes_{R} F \subset V_{\lambda}$ must be a connected component because $\operatorname{dim}\left(\mathbb{P}_{F}^{n}\right)=\operatorname{dim}\left(V_{\lambda}\right)=n$ and $V_{\lambda}$ is smooth. Finally, the conormal bundle of $\mathfrak{Z} \otimes_{R} F$ in $V$ is trivial because $\Psi$ is flat (since it is smooth). Using Corollary 3.4, we thus obtain an isomorphism

$$
\left.\omega_{\mathbb{P}_{F}^{n}} \simeq \omega_{V}\right|_{\mathbb{P}_{F}^{n}} \simeq \mathscr{O}(d(p-1)-n-1) .
$$

This gives a contradiction since $\omega_{\mathbb{P}_{F}^{n}} \simeq \mathscr{O}(-n-1)$.

\section{Cohomology and lattice points}

We now assume that the polynomials $Q_{0}, \ldots, Q_{n} \in k\left[T_{0}, \ldots, T_{n}\right]$ used to define our Moret-Bailly family $Y=\left(A \times \mathbb{P}^{n}\right) / H$ have degree $d=1$. After applying an automorphism of the projective $n$-space, we reduce to the situation $Q_{i}=T_{i}$. Tensoring the Euler sequence

$$
0 \longrightarrow \mathscr{O}_{\mathbb{P} n}(-1) \longrightarrow \mathscr{O}_{\mathbb{P}^{n}}^{\oplus n+1} \longrightarrow \mathscr{E}_{1} \longrightarrow 1
$$

with $\mathscr{O}_{\mathbb{P} n}(1)$ shows $\mathscr{E}_{1}=\Theta_{\mathbb{P} n}(-1)$, and the dual becomes $\mathscr{E}_{-1}=\Omega_{\mathbb{P}^{n}}^{1}(1)$. We now use the Bott formula

$$
h^{s}\left(\Omega_{\mathbb{P}^{n}}^{r}(l)\right)= \begin{cases}\left(\begin{array}{c}
n+l-r \\
n
\end{array}\right)\left(\begin{array}{c}
l-1 \\
r
\end{array}\right) & \text { if } s=0 \text { and } 0 \leqslant r \leqslant n, \\
\left(\begin{array}{c}
r-l \\
r
\end{array}\right)\left(\begin{array}{c}
-l-1 \\
n-r
\end{array}\right) & \text { if } s=n \text { and } 0 \leqslant r \leqslant n, \\
1 & \text { if } 0 \leqslant r=s \leqslant n \text { and } l=0, \\
0 & \text { else }\end{cases}
$$

to understand the groups $H^{i}\left(Y, \mathscr{O}_{Y}\right)$ better (compare with [Bot57, Section 4]); for an algebraic proof, see [Dol82, Section 2.3.2] or [Hua01, Section 4]. Note that the binomial coefficient $\left(\begin{array}{l}x \\ n\end{array}\right)=$ $x(x-1) \cdots(x-r+1) / n$ ! is defined for natural numbers $n$ and ring elements $x \in R$ whenever the denominator $n$ ! is invertible.

Our computation crucially relies on the splitting type of the Frobenius push-forward $F_{*}\left(\mathscr{O}_{\mathbb{P}} n\right)$, which indeed splits by the Horrocks criterion (see [OSS80, Section 2.3]). Understanding the splitting type involves seemingly innocent lattice point counts: For each integer $t$ and $l$, define multiplicities $\mu_{t, l} \geqslant 0$ as the number of lattice points $\left(l_{0}, \ldots, l_{n}\right) \in \mathbb{Z}^{n+1}$ contained in the polytope 
$P_{t, l} \subset \mathbb{R}^{n+1}$ defined by

$$
l_{0}+\cdots+l_{n}=t-p l \text { and } 0 \leqslant l_{0}, \ldots, l_{n} \leqslant p-1 .
$$

This is the intersection of an affine hyperplane with a hypercube. Clearly, the polytope is nonempty if and only if $0 \leqslant t-p l \leqslant(n+1)(p-1)$, and we have the recursion formula

$$
\mu_{t+p, l+1}=\mu_{t, l} \text {. }
$$

Note that the $\mu_{t, l}$ also depend on $n$ and $p$, but we neglect this dependence in notation. Applying a result of Achinger [Ach15, Theorem 2.1] to the toric variety $\mathbb{P}^{n}$, we get the following.

Proposition 6.1. The Frobenius push-forward $F_{*}\left(\mathscr{O}_{\mathbb{P}^{n}}(t)\right)$ splits as a sum of invertible sheaves, and the summand $\mathscr{O}_{\mathbb{P}^{n}}(l)$ appears with multiplicity $\mu_{t, l} \geqslant 0$. In other words, the splitting type is of the form

$$
(\underbrace{a, \ldots, a}_{\mu_{t, a}}, \ldots, \underbrace{b, \ldots, b}_{\mu_{t, b}})
$$

starting with $a=\lceil(t-(n+1)(p-1)) / p\rceil$ and ending with $b=\lfloor t / p\rfloor$.

In preparation for our analysis of $H^{i}\left(Y, \mathscr{O}_{Y}\right)$, we now express the cohomological invariants of certain locally free sheaves on $\mathbb{P}^{n}$ in terms of lattice points and binomial coefficients.

Proposition 6.2. The cohomological invariants of the locally free sheaf $\mathscr{F}_{r, t}=\Lambda^{r}\left(F^{*}\left(\Omega_{\mathbb{P}^{n}}^{1}(1)\right)\right)$ $\otimes \mathscr{O}_{\mathbb{P}^{n}}(t)$ are given by the formula

$$
h^{s}\left(\mathscr{F}_{r, t}\right)= \begin{cases}\sum_{l} \mu_{t, l}\left(\begin{array}{c}
n+l \\
n
\end{array}\right)\left(\begin{array}{c}
l+r-1 \\
r
\end{array}\right) & \text { if } s=0, \\
\sum_{l} \mu_{t, l}\left(\begin{array}{c}
-l \\
r
\end{array}\right)\left(\begin{array}{c}
-r-l-1 \\
n-r
\end{array}\right) & \text { if } s=n, \\
\mu_{t,-r} & \text { if } s=r, \\
0 & \text { else. }\end{cases}
$$

In particular, we have $h^{0}\left(\mathscr{F}_{r, t}\right)=0$ for $0 \leqslant t \leqslant p-1$ and $h^{s}\left(\mathscr{F}_{0,0}\right)=0$ for $s \geqslant 1$.

Proof. Set $\mathscr{F}=\mathscr{F}_{r, t}$. We have $H^{s}\left(\mathbb{P}^{n}, \mathscr{F}\right)=H^{s}\left(\mathbb{P}^{n}, F_{*} \mathscr{F}\right)$ because the Frobenius map is affine, and the projection formula gives $F_{*} \mathscr{F}=\Omega_{\mathbb{P}^{n}}^{r}(r) \otimes F_{*}\left(\mathscr{O}_{\mathbb{P}^{n}}(t)\right)$. Now combine the Bott formula for $h^{s}\left(\Omega_{\mathbb{P}^{n}}^{r}(l)\right)$ and Achinger's description of $F_{*}\left(\mathscr{O}_{\mathbb{P}^{n}}(t)\right)$ to get the general formula for $h^{s}(\mathscr{F})$. In the special case $s=0$, this reduces to

$$
h^{0}\left(\mathscr{F}_{r, t}\right)=\sum_{l} \mu_{t, l}\left(\begin{array}{c}
n+l \\
n
\end{array}\right)\left(\begin{array}{c}
r+l-1 \\
r
\end{array}\right) .
$$

The second binomial coefficient vanishes for $l \leqslant 0$. For $l \geqslant 1$ and $t \leqslant p-1$, the multiplicity $\mu_{t, l}$ is zero because the polytope $P_{t, l} \subset \mathbb{R}^{n+1}$ in (6.1) becomes empty. Finally, we have $\mathscr{F}_{0,0}=\mathscr{O}_{\mathbb{P} n}$ and thus $h^{s}\left(\mathscr{F}_{0,0}\right)=0$ in all degrees $s \geqslant 1$.

We now can express the cohomology groups of our Moret-Bailly family as follows.

Proposition 6.3. Suppose $p \geqslant n+1$. For every degree $i \geqslant 0$, the Leray-Serre spectral sequence for $\varphi: Y \rightarrow \mathbb{P}^{n}$ gives a natural identification

$$
H^{i}\left(Y, \mathscr{O}_{Y}\right)= \begin{cases}H^{j}\left(\mathbb{P}^{n}, \Lambda^{j}\left(\mathscr{E}_{-p}\right)\right) & \text { if } i=2 j \text { is even } \\ H^{j}\left(\mathbb{P}^{n}, \Lambda^{j}\left(\mathscr{E}_{-p}\right) \otimes \mathscr{O}_{\mathbb{P}^{n}}(1)\right) & \text { if } i=2 j+1 \text { is odd }\end{cases}
$$




\section{RÖSSLER AND S. SCHRÖER}

Moreover, the dimensions of these vector spaces are given by the formula

$$
h^{i}\left(\mathscr{O}_{Y}\right)= \begin{cases}\mu_{0,-j} & \text { if } i=2 j \text { is even, } \\ \mu_{1,-j} & \text { if } i=2 j+1 \text { is odd },\end{cases}
$$

where $\mu_{t, l} \geqslant 0$ is the number of lattice points in the polytope $P_{t, l} \subset \mathbb{R}^{n+1}$ as in (6.1).

Proof. The assertion indeed holds for $i=0$ because $\varphi_{*}\left(\mathscr{O}_{Y}\right)=\mathscr{O}_{\mathbb{P}}=\Lambda^{0}\left(\mathscr{E}_{-p}\right)$, and the only lattice point in $P_{0,0}$ has coordinates $l_{0}=\cdots=l_{n}=0$. Suppose from now on that $i \geqslant 1$.

The Leray-Serre spectral sequence is $E_{2}^{i, j}=H^{i}\left(\mathbb{P}^{n}, R^{j} \varphi_{*} \mathscr{O}_{Y}\right) \Rightarrow H^{i+j}\left(Y, \mathscr{O}_{Y}\right)$. For dimension reasons, the differentials $d_{r}: E_{r}^{i, j} \rightarrow E_{r}^{i+r, j-r+1}$ vanish on the $E_{r}$-pages whenever $r \geqslant n+1$. Since $p-1 \geqslant n$, they must also vanish on the pages with $2 \leqslant r \leqslant n$, according to Proposition 2.3. In turn, the associated graded on the abutment is

$$
\operatorname{gr} H^{i}\left(Y, \mathscr{O}_{Y}\right)=\bigoplus_{r+s=i} H^{s}\left(\mathbb{P}^{n}, \Lambda^{r}\left(R^{1} \varphi_{*}\left(\mathscr{O}_{Y}\right)\right)\right) .
$$

In our situation, $R^{1} \varphi_{*}\left(\mathscr{O}_{Y}\right)=\mathscr{O}_{\mathbb{P}^{n}}(1) \oplus \mathscr{E}_{-p}=F^{*}\left(\Omega^{1}(1)\right) \oplus \mathscr{O}_{\mathbb{P}^{n}}(1)$ by Proposition 3.2, which shows $\Lambda^{r}\left(R^{1} \varphi_{*}\left(\mathscr{O}_{Y}\right)\right)=\mathscr{F}_{r, 0} \oplus \mathscr{F}_{r-1,1}$ in the notation of Proposition 6.2 . Here we set $\mathscr{F}_{-1,1}=0$ for convenience. Thus

$$
h^{i}\left(\mathscr{O}_{Y}\right)=\sum_{s=0}^{i} h^{s}\left(\mathscr{F}_{i-s, 0}\right)+\sum_{s=0}^{i} h^{s}\left(\mathscr{F}_{i-s-1,1}\right) .
$$

The summands for $s=0$ vanish by Proposition 6.2. Furthermore, for $1 \leqslant s \leqslant n-1$, we have

$$
s \neq i-s \Rightarrow h^{s}\left(\mathscr{F}_{i-s, 0}\right)=0 \quad \text { and } \quad s \neq i-s-1 \Rightarrow h^{s}\left(\mathscr{F}_{i-s-1,1}\right)=0 .
$$

In the boundary case $i=n$, the last summands $h^{n}\left(\mathscr{F}_{0,0}\right)$ and $h^{n}\left(\mathscr{F}_{-1,1}\right)$ vanish, the former by Proposition 6.2, the latter because $\mathscr{F}_{-1,1}=0$. In turn, the sum (6.4) simplifies to

$$
h^{i}\left(\mathscr{O}_{Y}\right)= \begin{cases}h^{j}\left(\mathscr{F}_{j, 0}\right) & \text { if } i=2 j \text { is even }, \\ h^{j}\left(\mathscr{F}_{j, 1}\right) & \text { if } i=2 j+1 \text { is odd } .\end{cases}
$$

The formula for the vector space dimensions now follows from Proposition 6.2. We also see that the filtration on the abutment $H^{i}\left(Y, \mathscr{O}_{Y}\right)$ has merely one step, which gives the natural identification (6.3) of groups.

Because of the relevance for the Picard scheme, we record the following.

Corollary 6.4. For every $p>0$ and every $n \geqslant 0$, we have $h^{1}\left(\mathscr{O}_{Y}\right)=n+1$, whereas $h^{2}\left(\mathscr{O}_{Y}\right)$ equals the number of lattice points $\left(l_{0}, \ldots, l_{n}\right)$ satisfying $l_{0}+\cdots+l_{n}=p$ and $0 \leqslant l_{0}, \ldots, l_{n} \leqslant p-1$.

Proof. One easily checks that the terms $H^{i}\left(\mathbb{P}^{n}, \varphi_{*}\left(\mathscr{O}_{Y}\right)\right)$ for $i \geqslant 1$ and also the term $H^{3}\left(\mathbb{P}^{n}\right.$, $\left.R^{1} \varphi_{*}\left(\mathscr{O}_{Y}\right)\right)$ on the $E_{2}$-page for the Leray-Serre spectral sequence with respect to $\varphi: Y \rightarrow \mathbb{P}^{n}$ vanish. In turn, the formula for $h^{1}\left(\mathscr{O}_{Y}\right)$ and $h^{2}\left(\mathscr{O}_{Y}\right)$ of Proposition 6.3 hold regardless of the assumption $p \geqslant n+1$. In particular, $h^{1}\left(\mathscr{O}_{Y}\right)=n+1$ because the only lattice points in the polytope $P_{1,0} \subset \mathbb{R}^{n+1}$ are the standard basis vectors.

We now consider the case that gives varieties with $c_{1}=0$, which means

$$
n=p-2, \quad \operatorname{dim}(Y)=2 n+1=2 p-3, \quad \text { and } \quad \omega_{Y}=\mathscr{O}_{Y} .
$$




\section{MoRet-BAILly FAMILIES AND NON-LIFTABLE SCHEMES}

With computer algebra [BCFS10], we computed the cohomological invariants for the first six primes in the following table:

\begin{tabular}{llll}
\hline$p$ & $n$ & $\operatorname{dim}(Y)$ & $h^{0}\left(\mathscr{O}_{Y}\right), \ldots, h^{n}\left(\mathscr{O}_{Y}\right)$ \\
\hline 2 & 0 & 1 & 1 \\
\hline 3 & 1 & 3 & 1,2 \\
\hline 5 & 3 & 7 & $1,4,52,68$ \\
\hline 7 & 5 & 11 & $1,6,786,1251,6891,7872$ \\
\hline 11 & 9 & 19 & $1,10,167950,293830,18480520,25109950$, \\
& & & $251849140,296659645,859743835,905642810$ \\
\hline 13 & 11 & 23 & $1,12,2496132,4457256,825038490,1149834280,27258578260$, \\
& & & $33480335274,223425722070,250522227132,616161367152$, \\
& & 639330337978
\end{tabular}

The running time for $h^{i}\left(\mathscr{O}_{Y}\right)$ at the prime $p=13$ and in degree $i=12$ was about three days.

\section{Cohomology and weights}

The goal of this section is to gain further control over the cohomology of the Moret-Bailly family $Y=\left(A \times \mathbb{P}^{n}\right) / H$, in particular for $H^{2}\left(Y, \mathscr{O}_{Y}\right)$ and $H^{1}\left(Y, \Theta_{Y}\right)$, by using automorphisms of the superspecial abelian variety $A$ and their induced representations on cohomology. This is best formulated with the machinery of weights and requires some preparation. Fix some integer $l \geqslant 1$, and consider

$$
G=\mu_{l}=\mathbb{G}_{m}[l]=\operatorname{Spec} \mathbb{Z}[T] /\left(T^{l}-1\right),
$$

viewed as a family of finite diagonalizable group schemes over the ground ring $R=\mathbb{Z}$. Let $S$ be a scheme endowed with trivial $G$-action. Recall that by [DG70a, Exposé I, Proposition 4.7.3], a $G$-linearization for a quasicoherent sheaf $\mathscr{F}$ on $S$ is nothing but a weight decomposition $\mathscr{F}=$ $\bigoplus_{w} \mathscr{F}_{w}$, where $w$ runs over the character group $\mathbb{Z} / l \mathbb{Z}=\operatorname{Hom}\left(G, \mathbb{G}_{m}\right)$. Such characters are also known as weights. A weight $w$ is called trivial if $\mathscr{F}_{w}=0$. We say that the sheaf $\mathscr{F}$ is pure of weight $w_{0}$ if all other weights $w \neq w_{0}$ are trivial.

For each ring $A$, the group elements $\zeta \in G(A) \subset A^{\times}$act on $\mathscr{F}_{w} \otimes_{R} A$ via multiplication by $\lambda=w(\zeta)$. Note that for every base change $a: \operatorname{Spec}(k) \rightarrow S$ for some field $k$ containing a primitive $l$ th root of unity $\zeta$, the weight decomposition for $\mathscr{F}$ becomes the eigenspace decomposition on the vector space $V=\mathscr{F}(a)$ for the automorphism $\zeta: V \rightarrow V$ with respect to the eigenvalues $\lambda=w(\zeta)$. Also note that $G$-linearizations for the sheaf $\mathscr{F}$ correspond to $G$-actions on the finite $S$-scheme stemming from the sheaf of dual numbers $\mathscr{A}=\mathscr{O}_{S} \oplus \mathscr{F}$ or the vector $S$-scheme coming from $\mathscr{A}=\operatorname{Sym}^{\bullet}(\mathscr{F})$.

Now assume that we are over a ground field $k$ of characteristic $p>0$. Since $\mu_{l}$ is already defined over $\mathbb{F}_{p}$, we have a canonical identification $\mu_{l}^{(p)}=\mu_{l}$, and the relative Frobenius map $\mu_{l} \rightarrow \mu_{l}^{(p)}=\mu_{l}$ given by $\zeta \mapsto \zeta^{p}$ induces multiplication by $p$ on the character group. In turn, the Frobenius pullback $\mathscr{F}^{(p)}$ has two canonical $G$-linearizations: one stemming from $\mu_{l}^{(p)}=\mu_{l}$ with $\left(\mathscr{F}^{(p)}\right)_{w}=\left(\mathscr{F}_{w}\right)^{(p)}$, the other via the Frobenius map, such that $\left(\mathscr{F}^{(p)}\right)_{p w}=\left(\mathscr{F}_{w}\right)^{(p)}$. It turns 


\section{RÖSSLER AND S. SCHRÖER}

out that the latter is more important for us.

In what follows, we assume that $G=\mu_{l}$ acts on an abelian variety $A$ of dimension $g \geqslant 1$ so that there is a $\mu_{l}$-equivariant principal polarization $A \rightarrow P$ for the dual abelian variety $P=\operatorname{Pic}_{A / k}^{0}$ with the induced $\mu_{l}$-action. Note that the latter indeed can be achieved by passing to $B=(A \oplus P)^{\oplus 4}$ and making a finite field extension [KR03, Lemma 3.2]. Furthermore, we assume that the induced representation on $\operatorname{Lie}(A)$ is pure of weight $w_{0} \in \mathbb{Z} / l \mathbb{Z}$. Using the equivariant bijections $H^{1}\left(A, \mathscr{O}_{A}\right)=\operatorname{Lie}(P) \rightarrow \operatorname{Lie}(A)$, we immediately obtain the following.

Lemma 7.1. The induced $\mu_{l}$-representation on $H^{1}\left(A, \mathscr{O}_{A}\right)$ is also pure of weight $w=w_{0}$.

Now suppose additionally that $A$ is superspecial, and consider the Moret-Bailly family $Y=$ $\left(A \times \mathbb{P}^{n}\right) / H$ formed with the homogeneous polynomials $Q_{i}=T_{i}$ of degree $d=1$, as in Section 6 . Since the induced action of $G=\mu_{l}$ on $\operatorname{Lie}(A)$ is pure, each copy $\alpha_{p} \subset A$ is normalized by $G$, and we get induced actions on the quotients $A / \alpha_{p}$.

Consider the diagonal $G$-action on $X=A \times \mathbb{P}^{n}$, with trivial action on the second factor. This can also be seen as an action of the relative group scheme $G \times \mathbb{P}^{n}=\mu_{l, \mathbb{P} n}$ that normalizes the action of the family $H \subset X$ of height one group schemes with $\operatorname{Lie}_{H / \mathbb{P} n}=\mathscr{O}_{\mathbb{P} n}(-1)$ and thus induces a $G$-action on our Moret-Bailly family $Y=X / H$. The structure morphism $\varphi: Y \rightarrow \mathbb{P}^{n}$ is equivariant, with trivial $G$-action on the base. By Proposition 1.2, we have a four-term exact sequence

$$
0 \longrightarrow \mathscr{O}_{\mathbb{P}^{n}}(-1) \longrightarrow \operatorname{Lie}(A) \otimes_{k} \mathscr{O}_{\mathbb{P}^{n}} \longrightarrow \operatorname{Lie}_{Y / k} \longrightarrow \mathscr{O}_{\mathbb{P}^{n}}(-p) \longrightarrow 0
$$

The cokernel for the inclusion on the left is the sheaf $\mathscr{E} 1$, and the above yields the short exact sequence $0 \rightarrow \mathscr{E}_{1} \rightarrow \operatorname{Lie}_{Y / k} \rightarrow \mathscr{O}_{\mathbb{P}^{n}}(-p) \rightarrow 0$ of sheaves with $G$-linearizations. We saw in the proof for Proposition 3.2 that $\operatorname{Ext}^{1}\left(\mathscr{O}_{\mathbb{P}}(-p), \mathscr{E}_{1}\right)=0$. By [DG70a, Exposé I, Proposition 4.7.4], we may choose a splitting that respects linearizations. In turn, $\operatorname{Lie}_{Y / \mathbb{P}^{n}}=\mathscr{E}_{1} \oplus \mathscr{O}_{\mathbb{P}}(-p)$ as sheaves with $G$-linearization.

LEMma 7.2. In the above setting, the summand $\mathscr{E}_{1}$ is pure of weight $w=w_{0}$, whereas $\mathscr{O}_{\mathbb{P}^{n}}(-p)$ is pure of weight pwo.

Proof. The sheaf $\operatorname{Lie}(A) \otimes_{k} \mathscr{O}_{\mathbb{P}^{n}}$ is pure of weight $w=w_{0}$; hence the same holds for the subsheaf $\mathscr{O}_{\mathbb{P}^{n}}(-1)$ and the quotient sheaf $\mathscr{E} 1$. The relative Frobenius map $A \rightarrow A^{(p)}$ becomes $G$-equivariant, provided that we take the induced $G$-action via the Frobenius map $\mu_{l} \rightarrow \mu_{l}^{(p)}=\mu_{l}$. In turn, $\mathscr{O}_{\mathbb{P}^{n}}(-p) \subset \operatorname{Lie}\left(A^{(p)}\right) \otimes_{k} \mathscr{O}_{\mathbb{P}^{n}}$ is pure of weight $w=p w_{0}$.

We now make a similar analysis for the higher direct images $R^{i} \varphi_{*}\left(\mathscr{O}_{Y}\right)$, which also come with induced $G$-linearizations. To understand their weight decompositions, it suffices to treat the case $i=1$, according to Proposition 2.3. Now we use the canonical projection $Y=\left(A \times \mathbb{P}^{n}\right) / H \rightarrow$ $A / A[F] \times \mathbb{P}^{n}=A^{(p)} \times \mathbb{P}^{n}$. This map becomes $G$-equivariant if the right-hand side is endowed with the $\mu_{l}$-action coming from the Frobenius map.

We already saw in Proposition 3.2 that we have an exact sequence of coherent sheaves $0 \rightarrow$ $\mathscr{O}_{\mathbb{P}^{n}}(1) \rightarrow R^{1} \varphi_{*}\left(\mathscr{O}_{Y}\right) \rightarrow \mathscr{E}_{-p} \rightarrow 0$ and that all such extension split. As above, we see that there is a direct sum decomposition $R^{1} \varphi_{*}\left(\mathscr{O}_{Y}\right)=\mathscr{O}_{\mathbb{P}^{n}}(1) \oplus \mathscr{E}_{-p}$ of sheaves with $G$-linearizations.

LEMma 7.3. In the above setting, the summand $\mathscr{O}_{\mathbb{P}^{n}}(1)$ is pure of weight $w=p w_{0}$, whereas $\mathscr{E}_{-p}$ is pure of weight $p^{2} w_{0}$.

Proof. The Lie algebra $\mathfrak{a}=\operatorname{Lie}(A)$ is pure of weight $w=w_{0}$. According to Lemma 7.1, the same holds for $H^{1}\left(A, \mathscr{O}_{A}\right)$. In turn, the Frobenius pullback $H^{1}\left(A, \mathscr{O}_{A}\right)^{(p)}$ is pure of weight $w=p w_{0}$ 


\section{Moret-BAILly FAMILIES AND NON-LIFTABLE SCHEMES}

since we use the action stemming from the Frobenius map $\mu_{l} \rightarrow \mu_{l}^{(p)}$. We now proceed as for Lemma 7.2.

The $G$-action on the Moret-Bailly family $Y$ induces $G$-representations on Hodge groups $H^{s}\left(Y, \Omega_{Y}^{r}\right)$ and the tangent cohomology $H^{s}\left(Y, \Theta_{Y}\right)$. Since the $G$-action respects the morphism $\varphi: Y \rightarrow \mathbb{P}^{n}$, we also have induced representations on the groups $H^{s}\left(Y, \varphi^{*} \Theta_{\mathbb{P} n}\right)$ and $H^{s}\left(Y, \Theta_{Y / \mathbb{P}^{n}}\right)$. We now compute the weights in some relevant cases.

Proposition 7.4. Suppose $(p, n) \neq(2,1)$. With respect to the induced $G$-representation, the following hold:

(i) The group $H^{2}\left(Y, \mathscr{O}_{Y}\right)$ is pure of weight $w=p^{2} w_{0}$.

(ii) For the cohomology group $H^{1}\left(Y, \Theta_{Y / \mathbb{P}^{n}}\right)$, the only possible non-trivial weights are of the form $w=m w_{0}$ with coefficient $m \in\left\{p+1, p^{2}+1, p^{2}+p\right\}$.

(iii) For $H^{1}\left(Y, \varphi^{*} \Theta_{Y}\right)$, the only possible non-trivial weights are of the form $w=m w_{0}$ with $m \in\left\{p, p^{2}\right\}$.

Proof. According to Proposition 6.3, the Leray-Serre spectral sequence for the morphism $\varphi: Y \rightarrow$ $\mathbb{P}^{n}$ induces a canonical identification $H^{2}\left(Y, \mathscr{O}_{Y}\right)=H^{1}\left(\mathbb{P}^{n}, \mathscr{E}_{-p}\right)$. By Lemma 7.3 , the sheaf $\mathscr{E}_{-p}$ is pure of weight $w=p^{2} w_{0}$, and assertion (i) follows.

Next we compute the weights in $H^{1}\left(Y, \Theta_{Y / \mathbb{P}^{n}}\right)$. The projection formula for $\Theta_{Y / \mathbb{P}^{n}}=\varphi^{*} \operatorname{Lie}_{Y / \mathbb{P}^{n}}$ gives $R^{i} \varphi_{*}\left(\Theta_{Y / \mathbb{P}^{n}}\right)=\operatorname{Lie}_{Y / \mathbb{P}^{n}} \otimes R^{i} \varphi_{*}\left(\mathscr{O}_{Y}\right)$, and the Leray-Serre spectral sequence yields an exact sequence

$$
0 \longrightarrow H^{1}\left(\mathbb{P}^{n}, \operatorname{Lie}_{Y / \mathbb{P}^{n}}\right) \longrightarrow H^{1}\left(Y, \Theta_{Y / \mathbb{P}^{n}}\right) \longrightarrow H^{0}\left(\mathbb{P}^{n}, \operatorname{Lie}_{Y / \mathbb{P}^{n}} \otimes R^{1} \varphi_{*}\left(\mathscr{O}_{Y}\right)\right) .
$$

Recall from Proposition 3.2 that

$$
\operatorname{Lie}_{Y / \mathbb{P}^{n}}=\mathscr{E}_{1} \oplus \mathscr{O}_{\mathbb{P}^{n}}(-p) \quad \text { and } \quad R^{1} \varphi_{*}\left(\mathscr{O}_{Y}\right)=\mathscr{O}_{\mathbb{P}^{n}}(1) \oplus \mathscr{E}_{-p}
$$

One easily computes $H^{r}\left(\mathbb{P}^{n}, \mathscr{E}_{1}\right)=0$ for all $r \geqslant 1$ using the short exact sequence $0 \rightarrow \mathscr{O}_{\mathbb{P}^{n}}(-1) \rightarrow$ $\mathscr{O}_{\mathbb{P} n}^{\oplus n+1} \rightarrow \mathscr{E}_{1} \rightarrow 0$. Furthermore, $H^{1}\left(\mathbb{P}^{n}, \mathscr{O}_{\mathbb{P} n}(-p)\right)$ is non-zero only if $n=1$ and $p=2$, which was excluded. In turn, it suffices to understand the possible weights in $H^{0}\left(\mathbb{P}^{n}, \mathscr{F}\right)$ for the sheaf $\mathscr{F}=\operatorname{Lie}_{Y / \mathbb{P} n} \otimes R^{1} \varphi_{*}\left(\mathscr{O}_{Y}\right)$. For this we compute the weights occurring in each of the summands

$$
\mathscr{E}_{1} \otimes \mathscr{O}_{\mathbb{P}^{n}}(1), \quad \mathscr{O}_{\mathbb{P}^{n}}(-p) \otimes \mathscr{O}_{\mathbb{P}^{n}}(1), \quad \mathscr{E}_{1} \otimes \mathscr{E}_{-p}, \quad \text { and } \quad \mathscr{O}_{\mathbb{P}^{n}}(-p) \otimes \mathscr{E}_{-p}
$$

inside $\mathscr{F}$. According to Lemmas 7.2 and 7.3 , these are pure of weight $w=m w_{0}$, where the coefficient $m$ is an integer of the form $1+p, p+p, 1+p^{2}$, and $p+p^{2}$, respectively. The second case does not contribute because the global sections for $\mathscr{O}_{\mathbb{P}^{n}}(1-p)$ vanish. Assertion (ii) follows.

It remains to understand the weights in $H^{1}\left(Y, \varphi^{*} \Theta_{\mathbb{P}^{n}}\right)$. Now the projection formula gives $R^{i} \varphi_{*}\left(\varphi^{*} \Theta_{\mathbb{P}^{n}}\right)=\Theta_{\mathbb{P}^{n}} \otimes R^{i} \varphi_{*}\left(\mathscr{O}_{Y}\right)$, and the Leray-Serre spectral sequence yields an exact sequence

$$
0 \longrightarrow H^{1}\left(\mathbb{P}^{n}, \Theta_{\mathbb{P}^{n}}\right) \longrightarrow H^{1}\left(Y, \varphi^{*} \Theta_{\mathbb{P}^{n}}\right) \longrightarrow H^{0}\left(\mathbb{P}^{n}, \Theta_{\mathbb{P}^{n}} \otimes R^{1} \varphi_{*}\left(\mathscr{O}_{Y}\right)\right) \text {. }
$$

From the Euler sequence $0 \rightarrow \mathscr{O}_{\mathbb{P}} \rightarrow \mathscr{O}_{\mathbb{P} n}^{\oplus n+1}(1) \rightarrow \Theta_{\mathbb{P} n} \rightarrow 0$, one easily infers that the term on the left vanishes. As above, it suffices to understand the weights in $H^{0}\left(\mathbb{P}^{n}, \mathscr{F}\right)$ for the coherent sheaf $\mathscr{F}=\Theta_{\mathbb{P}^{n}} \otimes R^{1} \varphi_{*}\left(\mathscr{O}_{Y}\right)$. This is the sum of $\Theta_{\mathbb{P}^{n}} \otimes \mathscr{O}_{\mathbb{P}^{n}}(1)$ and $\Theta_{\mathbb{P}^{n}} \otimes \mathscr{E}_{-p}$. Now the summands are pure of weight $w=m w_{0}$ with $m=0+p$ and $m=0+p^{2}$, which gives assertion (iii).

We now deduce a crucial fact that can be formulated without the machinery of weights. 


\section{RÖSSLER AND S. SCHRÖER}

Corollary 7.5. Suppose $p \neq 2$. Then the sign involution on the superspecial abelian variety $A$ induces an action of the multiplicative group $G=\{ \pm 1\}$ on our Moret-Bailly family $Y=(A \times$ $\left.\mathbb{P}^{n}\right) / H$, and the induced representation on the cohomology groups $H^{2}\left(Y, \mathscr{O}_{Y}\right)$ and $H^{1}\left(Y, \varphi^{*} \Theta_{Y}\right)$ is multiplication by $\lambda=-1$, whereas on $H^{1}\left(Y, \Theta_{Y / \mathbb{P}^{n}}\right)$ it is multiplication by $\lambda=1$.

Proof. We may assume that $k$ is algebraically closed and regard the abstract group $G=\{ \pm 1\}$ as the diagonalizable group scheme $G=\mu_{l}$ with $l=2$. Write $A=E_{1} \times \cdots \times E_{g}$ as a product of supersingular elliptic curve, and use the canonical identification $E_{i}=\mathrm{Pic}_{E_{i} / k}$ to obtain an equivariant principal polarization.

The induced $G$-representation on $\operatorname{Lie}(A)$ is multiplication by $\lambda=-1$. This is pure of weight $w_{0}=1$, seen as an element of the character group $\mathbb{Z} / 2 \mathbb{Z}$. As discussed above, we get an induced action on $Y$ such that the quotient map $\epsilon: A \times \mathbb{P}^{n} \rightarrow Y$ is equivariant. According to Proposition 7.4, the induced representation on $H^{2}\left(Y, \mathscr{O}_{Y}\right)$ is pure of weight $w=p^{2} w_{0}=1$ because $p \equiv 1$ modulo $l$. Similarly, $H^{1}\left(Y, \varphi^{*} \Theta_{Y}\right)$ has weight $w=1$. In contrast, the only possible non-zero weights on $H^{1}\left(Y, \Theta_{Y / \mathbb{P}^{n}}\right)$ are $w=m w_{0}$, with coefficient $m \equiv 0$ modulo $l$. In turn, $H^{1}\left(Y, \Theta_{Y / \mathbb{P}^{n}}\right)$ is pure of weight $w=0$.

\section{Non-existence of formal liftings}

We continue to study our Moret-Bailly families $Y=\left(A \times \mathbb{P}^{n}\right) / H$ over the ground field $k$ of characteristic $p>0$, formed with a superspecial abelian variety $A$ of dimension $g=n+1$ and the homogeneous polynomials $Q_{i}=T_{i}$ of degree $d=1$. The sign involution $a \mapsto-a$ on the abelian variety $A$ induces an action of the cyclic group $G=\{ \pm 1\}$ on the total space $Y$. For simplicity, the inclusion $G \subset \operatorname{Aut}(Y / k)$ is also called the sign involution. We now regard this $G$-action on $Y$ as additional structure and seek to understand its behavior under deformations.

Let $R$ be a complete local noetherian ring with residue field $R / \mathfrak{m}_{R}=k$ and $\mathfrak{Y} \rightarrow \operatorname{Spf}(R)$ be a proper flat morphism of formal schemes with closed fiber $Y=\mathfrak{Y} \otimes_{R} k$. We say that this morphism is projectively algebraizable if there is an invertible sheaf on $\mathfrak{Y}$ whose restriction to $Y$ is ample. According to Grothendieck's existence theorem, the flat formal $R$-scheme $\mathfrak{Y}$ then is the formal completion of some flat projective $R$-scheme [GD61, Theorem 5.4.5].

Proposition 8.1. Suppose $p \geqslant 3$. If the sign involution $G \subset \operatorname{Aut}(Y)$ extends to some $G \subset$ $\operatorname{Aut}(\mathfrak{Y} / R)$, then $\mathfrak{Y} \rightarrow \operatorname{Spf}(R)$ is projectively algebraizable.

The proof for Proposition 8.1 requires a bit of preparation and will be given below. Let us first apply the result: We say that the proper scheme $Y$ together with the sign involution formally lifts to characteristic zero if we may choose some $\mathfrak{Y} \rightarrow \operatorname{Spf}(R)$ and $G \subset \operatorname{Aut}(\mathfrak{Y} / R)$ as above, where furthermore the canonical map $\mathbb{Z} \rightarrow R$ is injective. Note that we may assume that $R$ is integral and one-dimensional, by passing to the residue class ring for some suitable prime ideal.

Also note that $A$, like any abelian variety, projectively lifts to characteristic zero, but for $g \geqslant 2$ there are formal liftings $\mathfrak{A} \rightarrow \operatorname{Spf}(R)$ to characteristic zero that are not algebraizable. The situation for Moret-Bailly families is completely different. From the above result, using Corollary 3.4 and Theorem 5.1, we immediately get the following.

Theorem 8.2. Suppose $p \geqslant 3$ and $p-1 \leqslant g$. Then the Moret-Bailly family $Y=\left(A \times \mathbb{P}^{n}\right) / H$ together with the sign involution does not formally lift to characteristic zero.

We now prepare for the proof of Proposition 8.1. Recall that a $G$-linearized invertible sheaf on $Y=\left(A \times \mathbb{P}^{n}\right) / H$ is an invertible sheaf $\mathscr{L}$, together with a $G$-linearization, that is, a $G$-action on 


\section{Moret-BAILly FAMILIES AND NON-LIFTABLE SCHEMES}

the line bundle $L=\operatorname{Spec}\left(\operatorname{Sym}^{\bullet}\left(\mathscr{L}^{\vee}\right)\right)$ such that the structure morphism $L \rightarrow Y$ is equivariant. Write $\operatorname{Pic}(Y, G)$ for the abelian group of isomorphism classes for $G$-linearized invertible sheaves. This can also be viewed as the equivariant cohomology group $H^{1}\left(Y, G ; \mathscr{O}_{Y}^{\times}\right)$or the Picard group of the quotient stack $[Y / G]$.

Now let $\mathfrak{Y} \rightarrow \operatorname{Spf}(R)$ be any formal deformation of the Moret-Bailly family $Y$ over some complete local noetherian ring $R$ with residue field $R / \mathfrak{m}_{R}=k$, and suppose that the sign involution extends to some $G \subset \operatorname{Aut}(\mathfrak{Y} / R)$.

Proposition 8.3. The restriction map $\operatorname{Pic}(\mathfrak{Y}, G) \rightarrow \operatorname{Pic}(Y, G)$ is surjective.

Proof. Refining the descending chain $\mathfrak{m}_{R}^{j}$, we get a descending chain of ideals $\mathfrak{a}_{i}$ with the property length $\left(\mathfrak{a}_{i} / \mathfrak{a}_{i+1}\right)=1$, such that $R=\lim R_{i}$, where $R_{i}=R / \mathfrak{a}_{i}$. Setting $Y_{i}=\mathfrak{Y} \otimes_{R} R_{i}$, we get an increasing sequence $Y=Y_{0} \subset Y_{1} \subset \cdots$, where each $Y_{i}$ is a proper flat $R_{i}$-scheme and the comparison maps $Y_{i-1} \rightarrow Y_{i} \otimes_{R_{i}} R_{i-1}$ are isomorphisms. In fact, the formal scheme $\mathfrak{Y}$ is nothing but the resulting inverse system $\left(Y_{i}\right)_{i \geqslant 0}$ of $R$-schemes.

Let $\mathscr{L}_{0}$ be a $G$-linearized invertible sheaf on $Y_{0}=Y$. We show by induction on $i \geqslant 0$ that it extends to $Y_{i}$. This is obvious for $i=0$. Now suppose that we have a linearized extension $\mathscr{L}_{i}$ on $Y_{i}$. The ideal sheaf $\mathscr{I}$ for the closed embedding $Y_{i} \subset Y_{i+1}$ has square zero and is isomorphic to $\mathscr{O}_{Y}$ by flatness. In turn, we have a short exact sequence $0 \rightarrow \mathscr{O}_{Y} \rightarrow \mathscr{O}_{Y_{i+1}}^{\times} \rightarrow \mathscr{O}_{Y_{i}}^{\times} \rightarrow 1$, which gives an exact sequence

$$
0 \longrightarrow H^{1}\left(Y, \mathscr{O}_{Y}\right) \longrightarrow \operatorname{Pic}\left(Y_{i+1}\right) \longrightarrow \operatorname{Pic}\left(Y_{i}\right) \stackrel{\partial}{\longrightarrow} H^{2}\left(Y, \mathscr{O}_{Y}\right) .
$$

By the naturality of cohomology, this sequence is equivariant with respect to the induced $G$ actions. The isomorphism class of $\mathscr{L}_{i}$ is $G$-invariant, so Corollary 7.5 gives $\partial\left(\mathscr{L}_{i}\right)=-\partial\left(\mathscr{L}_{i}\right)$. With $p \neq 2$ we infer that the obstruction $\partial\left(\mathscr{L}_{i}\right)$ vanishes; hence $\mathscr{L}_{i}$ extends to some invertible sheaf on $Y_{i+1}$.

It remains to choose an extension that admits a linearization. To achieve this, we use the results from [ST18], discussed in more details in the proof of Proposition 8.4 below. Let $L$ be the set of isomorphism classes for pairs $\left(\mathscr{L}^{\prime}, \varphi^{\prime}\right)$, where $\mathscr{L}^{\prime}$ is invertible on $Y_{i+1}$ and $\varphi^{\prime}: \mathscr{L}_{i} \rightarrow$ $\mathscr{L}^{\prime} \mid Y_{i}$ is an isomorphism, and define $T=H^{1}\left(Y, \mathscr{O}_{Y}\right)$. Then $L$ carries the structure of a $T$ torsor with group of operators $G$, giving a cohomology class $[L] \in H^{1}(G, T)$. This cohomology group vanishes because $|G|=2$ is relatively prime to the characteristic $p \geqslant 3$. In turn, we can choose a lifting $\mathscr{L}_{i+1}$ whose isomorphism class is $G$-fixed. The group of automorphisms of $\mathscr{L}_{i+1}$ restricting to the identify on $\mathscr{L}_{i}$ is the vector space $V=\operatorname{Hom}\left(\mathscr{L}_{i+1}, \mathscr{O}_{Y}\right)=\operatorname{Hom}\left(\mathscr{L}_{0}, \mathscr{O}_{Y}\right)$. According to [ST18, Theorem 1.2], the obstruction to the existence of a $G$-linearization lies in the cohomology group $H^{2}\left(G, \mathfrak{a}_{i} / \mathfrak{a}_{i+1} \otimes_{k} V\right)$, which again vanishes. Summing up, the linearized sheaf $\mathscr{L}_{i}$ extends to $Y_{i+1}$.

Proof of Proposition 8.1. Suppose that there is a formal lifting $\mathfrak{Y} \rightarrow \operatorname{Spf}(R)$ to characteristic zero such that the sign involution extends to some $G \subset \operatorname{Aut}(\mathfrak{Y} / R)$. Choose a very ample invertible sheaf $\mathscr{L}$ on $Y$. Replacing $\mathscr{L}$ with $\mathscr{L} \otimes \sigma^{*}(\mathscr{L})$, where $\sigma \in G$ is the generator, we may assume that the isomorphism class is $G$-invariant. According to [Gro57, Theorem 5.2.1], there is a spectral sequence

$$
H^{r}\left(G, H^{s}\left(Y, \mathscr{O}_{Y}^{\times}\right)\right) \Longrightarrow H^{r+s}\left(Y, G, \mathscr{O}_{Y}^{\times}\right)
$$

The resulting five-term exact sequence shows that the obstruction to the existence of a $G$ linearization on $\mathscr{L}$ lies in the elementary abelian group $H^{2}\left(G, k^{\times}\right)=k^{\times} / k^{2 \times}$. Thus $\mathscr{O}_{Y}(1)=$ $\mathscr{L}^{\otimes 2}$ can be endowed with a $G$-linearization and is very ample. 


\section{RÖSSLER AND S. SCHRÖER}

By Proposition 8.3, there is some invertible sheaf $\mathscr{O}_{\mathfrak{Y}}(1)$ restricting to $\mathscr{O}_{Y}(1)$. According to Grothendieck's existence theorem, $\mathfrak{Y}$ is the formal completion of some flat projective $R$ scheme [GD61, Theorem 5.4.5]. In turn, our Moret-Bailly family $Y$ admits a projective lift to characteristic zero.

Recall that the Moret-Bailly family $Y=\left(A \times \mathbb{P}^{n}\right) / H$ comes with an induced morphism $\varphi: Y \rightarrow \mathbb{P}^{n}$. It turns out that extending the sign involution is essentially the same as extending this morphism. Let $\mathfrak{Y} \rightarrow \operatorname{Spf}(R)$ be any formal deformation of the Moret-Bailly family $Y$ over some complete local noetherian ring $R$ with residue field $R / \mathfrak{m}_{R}=k$

Proposition 8.4. Suppose $p \geqslant 3$. Then the following two conditions are equivalent:

(i) The morphism $\varphi: Y \rightarrow \mathbb{P}^{n}$ extends to a morphism $\mathfrak{Y} \rightarrow \mathbb{P}_{R}^{n}$.

(ii) The sign involution $G \subset \operatorname{Aut}(Y / k)$ extends to an inclusion $G \subset \operatorname{Aut}(\mathfrak{Y} / R)$.

Proof. We start with the implication (i) $\Rightarrow$ (ii). Suppose that $\varphi: Y \rightarrow \mathbb{P}^{n}$ extends to a morphism, which we likewise call $\varphi: \mathfrak{Y} \rightarrow \mathbb{P}_{R}^{n}$. In order to extend the action of $G=\{ \pm 1\}$, we use Rim's results about equivariant structures on versal deformations [Rim80], in the form obtained in [ST18]. Choose a Cohen ring $\Lambda$ with residue field $k$. Recall that this is a complete discrete valuation ring with maximal ideal $\mathfrak{m}_{\Lambda}=p \Lambda$. Let $\left(\operatorname{Art}_{\Lambda}\right)$ be the category of local Artin rings $A$ over $\Lambda$ with residue field $k$ and $\mathscr{F} \rightarrow\left(\operatorname{Art}_{\Lambda}\right)^{\text {op }}$ be the category fibered in groupoids whose fiber category over $A$ comprises the pairs $\xi=(X, \alpha)$, where $X$ is a proper flat scheme over $\mathbb{P}_{R}^{n}$ and $\alpha: Y \rightarrow X \otimes_{R} k$ is an isomorphism. This is a deformation category in the sense of Talpo and Vistoli [TV13]. To conform with [ST18], the symbol $A$, which otherwise denotes our superspecial abelian variety, is here also used for local Artin rings; this should not cause any confusion.

Using the notation from the proof of Proposition 8.3, we write $R=\lim R_{i}$. We now show by induction on $i \geqslant 0$ that there are compatible inclusions $G \subset \operatorname{Aut}\left(Y_{i} / \mathbb{P}_{R_{i}}^{n}\right)$. For $i=0$, we choose the given action on $Y_{0}=Y$. Now suppose that we already have the action on $Y_{i}$. Using the notation from [ST18], set $A=R_{i}$ and $A^{\prime}=R_{i+1}$. Write $\xi \in \mathscr{F}(A)$ for the given family $Y_{i}=\mathfrak{Y} \otimes_{R} A$ over $\mathbb{P}_{A}^{n}$ and $\operatorname{Lif}\left(\xi, A^{\prime}\right)$ for the set of isomorphism classes $[f]$ of all cartesian morphisms $f: \xi \rightarrow \xi^{\prime}$ over the inclusion $\operatorname{Spec}(A) \subset \operatorname{Spec}\left(A^{\prime}\right)$, in the fibered category $\mathscr{F}$. This set comes with a $G$-action, namely $\sigma \cdot[f]=\left[f \circ \sigma^{-1}\right]$. The family $Y_{i+1}=\mathfrak{Y} \otimes_{R} A^{\prime}$ yields an element $\xi_{i+1}$ in $L=\operatorname{Lif}\left(\xi, A^{\prime}\right)$, which thus is non-empty. Furthermore, it carries the structure of a torsor for the tangent space $T=H^{1}\left(Y, \Theta_{Y / \mathbb{P}^{n}}\right)$ for the deformation category. It is also endowed with $G$ as group of operators, meaning that the action

$$
L \times T \longrightarrow L, \quad([f], t) \longmapsto[f] \cdot t
$$

satisfies ${ }^{\sigma}([f] \cdot t)={ }^{\sigma}[f] \cdot{ }^{\sigma} t$.

Now write $f: \xi \rightarrow \xi^{\prime}$ for the lifting corresponding to $Y_{i+1}$, and let $\sigma \in G$ be the generator. Since $\sigma: Y_{i} \rightarrow Y_{i}$ is compatible with the morphism $\varphi: Y_{i} \rightarrow \mathbb{P}_{R_{i}}^{n}$, the new class $\left[f \circ \sigma^{-1}\right]$ differs from the old class by some element $t \in T$; in other words, ${ }^{\sigma}[f]=[f] \cdot t$. Applying the involution to this equation and using that it acts trivially on $T$ by our weight computation in Proposition 7.5 , we get

$$
[f]={ }^{\sigma}([f] \cdot t)={ }^{\sigma}[f] \cdot{ }^{\sigma} t={ }^{\sigma}[f] \cdot t=[f] \cdot 2 t .
$$

This ensures $2 t=0$, and hence $t=0$ since we are in characteristic $p \neq 2$. In other words, $[f] \in L$ is $G$-fixed.

Consequently, the object $\xi^{\prime} \in \mathscr{F}\left(A^{\prime}\right)$ corresponding to $Y_{i+1}=\mathfrak{Y} \otimes_{R} A^{\prime}$ has $G$-fixed isomorphism class. By [ST18, Theorem 3.3], the obstruction for extending the $G$-action from $Y_{i}$ to $Y_{i+1}$ 


\section{Moret-BAilly fAMilies AND NON-LIFTABle SCHEMES}

lies in $H^{2}\left(G, \mathfrak{a}_{i} / \mathfrak{a}_{i+1} \otimes_{k} \operatorname{Aut}_{\xi_{0}}\left(\xi_{k[\epsilon]}\right)\right)$. This group vanishes because $p \neq 2$, and we conclude that the action on $Y_{i}$ extends to an action on $Y_{i+1}$ over $\mathbb{P}_{R_{i+1}}^{n}$.

We now establish the reverse implication (ii) $\Rightarrow(\mathrm{i})$. Suppose that we have $G \subset \operatorname{Aut}(\mathfrak{Y} / R)$. By induction on $i \geqslant 0$, we show that $Y_{i} \rightarrow \operatorname{Spec}\left(R_{i}\right)$ factors over $\mathbb{P}_{R_{i}}^{n}$ in a compatible way, so that $G$ acts over $\mathbb{P}_{R_{i}}^{n}$.

Suppose that we already have a factorization $Y_{i} \rightarrow \mathbb{P}_{R_{i}}^{n}$. This morphism must be flat, by the fiber-wise criterion for flatness [GD66, Theorem 11.3.10]. The obstruction to extending $Y_{i}$ over $\mathbb{P}_{R_{i+1}}^{n}$ is an element $\alpha \in H^{2}\left(Y, \Theta_{Y / \mathbb{P}^{n}}\right)$. The short exact sequence $0 \rightarrow \Theta_{Y / \mathbb{P}^{n}} \rightarrow \Theta_{Y} \rightarrow \varphi^{*} \Theta_{\mathbb{P}^{n}} \rightarrow 0$ induces an exact sequence

$$
H^{1}\left(Y, \varphi^{*} \Theta_{\mathbb{P}^{n}}\right) \longrightarrow H^{2}\left(Y, \Theta_{Y / \mathbb{P}^{n}}\right) \longrightarrow H^{2}\left(Y, \Theta_{Y}\right) .
$$

Since $Y_{i+1}$ extends $Y_{i}$ over $R_{i+1}$, the image of $\alpha$ in $H^{2}\left(Y, \Theta_{Y}\right)$ vanishes; hence our obstruction belongs to the image of $H^{1}\left(Y, \varphi^{*} \Theta_{\mathbb{P}^{n}}\right)$. Let $\sigma \in G$ be the generator. By the weight computation in Corollary 7.5, we must have $\sigma^{*}(\alpha)=-\alpha$. On the other hand, the functoriality of the obstruction gives $\sigma^{*}(\alpha)=\alpha$. Using $p \neq 2$, we conclude that the obstruction vanishes; thus we have an extension $Y_{i+1} \rightarrow \mathbb{P}_{R_{i+1}}^{n}$.

It remains to check that the $G$-action on $Y_{i+1}$ is over $\mathbb{P}_{R_{i+1}}^{n}$. In light of the implication (i) $\Rightarrow\left(\right.$ ii), it suffices to check that there is at most one extension of $G \subset \operatorname{Aut}\left(Y_{i} / R_{i}\right)$ to $G \subset$ $\operatorname{Aut}\left(Y_{i+1} / R_{i+1}\right)$. As explained in [ST18, Text below Proposition 1.1], any two such extensions differ by a homomorphism of $G$ into the vector space of automorphisms of $Y_{i+1}$ restricting to the identity on $Y_{i}$. Using $p \neq 2$, we see that such homomorphisms are trivial.

\section{Non-existence of liftings to Witt vectors}

Fix integers $n, d \geqslant 1$, and form the Moret-Bailly family $Y$ with some superspecial abelian variety $A$ of dimension $g=n+1$ over a field $k$ of characteristic $p>0$, as in Section 3. Recall that it comes with two morphisms $\varphi: Y \rightarrow \mathbb{P}^{n}$ and $\psi: Y \rightarrow A^{(p)}$. Finally, recall that $\operatorname{dim}(Y)=2 n+1$ and that $\omega_{Y}=\varphi^{*} \mathscr{O}_{\mathbb{P}^{n}}(m)$, where $m=d(p-1)-(n+1)$. Here $\omega_{Y}=\operatorname{det}\left(\Omega_{Y / k}^{1}\right)$ is the dualizing sheaf of $Y$.

We suspect that regardless of the values of $n$ and $d$, the scheme $Y$ does not lift to characteristic zero and perhaps also not to the ring $W_{2}(k)$ of Witt vectors of length two. Regarding $Y=Y_{n, d, p}$ also in dependence of the prime $p=\operatorname{char}(k)$, we will show the following.

Theorem 9.1. Fix $n \geqslant 2$ and $d \geqslant 1$. Suppose $n \not \equiv 2$ modulo 4 and that the ground field $k$ is perfect. Then the Moret-Bailly family $Y$ does not lift over the ring $W_{2}(k)$ for almost all primes $p>0$.

This is an application of the Deligne-Illusie result on Kodaira-Nakano-Akizuki vanishing in positive characteristics, together with a Hirzebruch-Riemann-Roch computation of certain Euler characteristics based on our knowledge of the tangent sheaf $\Theta_{Y}$. The proof requires some preparation and is given toward the end of the section.

Recall that the Chow ring with rational coefficients of the projective space is a truncated polynomial ring $\mathrm{CH}^{\bullet}\left(\mathbb{P}^{n}\right)=\mathbb{Q}[h] /\left(h^{n+1}\right)$, where $h \in \mathrm{CH}^{1}\left(\mathbb{P}^{n}\right)$ is the class of the invertible sheaf $\mathscr{O}_{\mathbb{P}^{n}}(1)$. Consider the formal power series

$$
Q(x)=\left(\frac{-d x}{1-e^{d x}}\right)^{-1}\left(\frac{-d p x}{1-e^{d p x}}\right)\left(\frac{x}{1-e^{-x}}\right)^{n+1} \in \mathbb{Q}[[x]] .
$$




\section{RÖSSLER AND S. SCHRÖER}

It induces an element $Q(h) \in \mathrm{CH}^{\bullet}\left(\mathbb{P}^{n}\right)$, which appears in the following computation.

Proposition 9.2. Let $\mathscr{E}$ and $\mathscr{F}$ be coherent sheaves on $A^{(p)}$ and $\mathbb{P}^{n}$, respectively. Then the coherent sheaf $\mathscr{M}=\psi^{*}(\mathscr{E}) \otimes \varphi^{*}(\mathscr{F})$ on the Moret-Bailly family $Y$ has Euler characteristic

$$
\chi(\mathscr{M})=p^{g-1} \chi(\mathscr{E}) \int_{\mathbb{P}^{n}} \operatorname{ch}(\mathscr{F}) Q(h) .
$$

Proof. We have $\chi(\mathscr{M})=\int_{Y} \operatorname{ch}(\mathscr{M}) \operatorname{td}\left(\Theta_{Y}\right)$ according to the Hirzebruch-Riemann-Roch theorem, where $\operatorname{ch}(\mathscr{M})$ is the Chern character of $\mathscr{M}$ and $\operatorname{td}\left(\Theta_{Y}\right)$ is the Todd class of the tangent sheaf [Fu198, Corollary 15.2.2]. Recall that Todd classes are multiplicative in short exact sequences, hence $\operatorname{td}\left(\Theta_{Y}\right)=\operatorname{td}\left(\Theta_{Y / \mathbb{P}^{n}}\right) \operatorname{td}\left(\varphi^{*} \Theta_{\mathbb{P}^{n}}\right)$. Using the decomposition $\Theta_{Y / \mathbb{P}^{n}}=\varphi^{*}\left(\mathscr{E}_{d} \oplus \mathscr{O}_{\mathbb{P}^{n}}(-d p)\right)$ from Proposition 3.2 and the exact sequences

$$
0 \longrightarrow \mathscr{O}_{\mathbb{P}^{n}}(-d) \longrightarrow \mathscr{O}_{\mathbb{P}^{n}}^{\oplus n+1} \longrightarrow \mathscr{E}_{d} \longrightarrow 0 \quad \text { and } \quad 0 \longrightarrow \mathscr{O}_{\mathbb{P}^{n}} \longrightarrow \mathscr{O}_{\mathbb{P}^{n}}^{\oplus n+1}(1) \longrightarrow \Theta_{\mathbb{P}^{n}} \longrightarrow 0,
$$

we infer that the Todd class $\operatorname{td}\left(\Theta_{Y}\right) \in \mathrm{CH}^{\bullet}(Y)$ is the pullback of

$$
Q(h)=\operatorname{td}\left(\mathscr{L}^{\otimes-d}\right)^{-1} \operatorname{td}\left(\mathscr{L}^{\otimes-d p}\right) \operatorname{td}(\mathscr{L})^{n+1} \in \mathrm{CH}^{\bullet}\left(\mathbb{P}^{n}\right)
$$

with respect to $\varphi: Y \rightarrow \mathbb{P}^{n}$. Here $\mathscr{L}=\mathscr{O}_{\mathbb{P}^{n}}(1)$, with first Chern class $h \in \mathrm{CH}^{2}\left(\mathbb{P}^{n}\right)$ and Todd class $\operatorname{td}(\mathscr{L})=h /\left(1-e^{-h}\right)$.

The morphism $\varphi: Y \rightarrow \mathbb{P}^{n}$ factors over the morphism $\psi \times \varphi: Y \rightarrow A^{(p)} \times \mathbb{P}^{n}$ to the product, which is locally free of rank $p^{g-1}$, and we have $\mathscr{M}=(\psi \times \varphi)^{*}\left(\operatorname{pr}_{1}^{*} \mathscr{E} \otimes \operatorname{pr}_{2}^{*} \mathscr{F}\right)$. The projection formula thus gives

$$
\int_{Y} \operatorname{ch}(\mathscr{M}) \psi^{*}(Q(h))=p^{g-1} \int_{A^{(p)} \times \mathbb{P}^{n}} \operatorname{ch}\left(\operatorname{pr}_{1}^{*} \mathscr{E} \otimes \operatorname{pr}_{2}^{*} \mathscr{F}\right) \operatorname{pr}_{2}^{*}(Q(h)) .
$$

Since Chern characters are natural and multiplicative in tensor products, the above can be written as

$$
p^{g-1} \int_{A^{(p)} \times \mathbb{P}^{n}} \operatorname{pr}_{1}^{*}(\operatorname{ch}(\mathscr{E})) \cdot \operatorname{pr}_{2}^{*}(\operatorname{ch}(\mathscr{F}) Q(h)) .
$$

By the functoriality of proper push-forwards and the projection formula, this coincides with

$$
p^{g-1} \int_{\mathbb{P}^{n}} \operatorname{pr}_{2 *}\left(\operatorname{pr}_{1}^{*}(\operatorname{ch}(\mathscr{E}))\right) \cdot \operatorname{ch}(\mathscr{F}) Q(h) .
$$

We now use that the Todd class for the tangent sheaf of any abelian variety is the unit element in the Chow group. Using this together with the compatibility of proper push-forwards with flat pullbacks [Ful98, Proposition 1.7] applied to the diagram

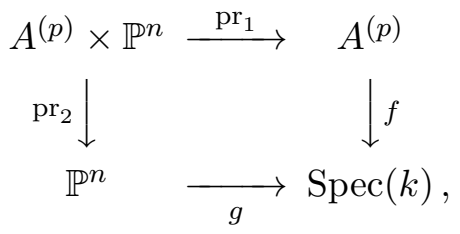

we see that $\operatorname{pr}_{2 *}\left(\operatorname{pr}_{1}^{*}(\operatorname{ch}(\mathscr{E}))\right)=g^{*} f_{*}\left(\operatorname{ch}(\mathscr{E}) \operatorname{td}\left(\Theta_{A^{(p)}}\right)\right)=\chi(\mathscr{E})$ in the Chow group $\mathrm{CH}^{\bullet}\left(\mathbb{P}^{n}\right)$. This yields the desired formula for the Euler characteristic of $\mathscr{M}$.

Now suppose that $\mathscr{E}$ is an ample invertible sheaf on $A^{(p)}$, and let $s \geqslant 1$ be an integer. Since $\psi \times \varphi: Y \rightarrow A^{(p)} \times \mathbb{P}^{n}$ is finite, the invertible sheaf $\mathscr{L}=\psi^{*}(\mathscr{E}) \otimes \varphi^{*}\left(\mathscr{O}_{\mathbb{P}^{n}}(s)\right)$ on the Moret-Bailly family $Y$ is ample. 


\section{Moret-BAILly FAMILIES AND NON-LIFTABLE SCHEMES}

Proposition 9.3. In the above setting, fix the integers $n \geqslant 2$ and $d \geqslant 1$ and some $s>\frac{1}{2}(n+d+1)$. Furthermore, assume $n \not \equiv 2$ modulo 4. Then the invertible sheaf $\mathscr{M}=\mathscr{L} \otimes \omega_{Y}$ has Euler characteristic $\chi(\mathscr{M})<0$ for almost all primes $p>0$.

Proof. We have $\mathscr{M}=\psi^{*}(\mathscr{E}) \otimes \varphi^{*}(\mathscr{F})$ with $\mathscr{F}=\mathscr{O}_{\mathbb{P}^{n}}(d(p-1)-(n+1)+s)$, by Proposition 3.4. Hence Proposition 9.2 gives

$$
\chi(\mathscr{M})=p^{g-1} \chi(\mathscr{E}) \int_{\mathbb{P}^{n}} Q(h) e^{(d p-d-n-1+s) h} .
$$

The factor $\chi(\mathscr{E})$ is strictly positive, by the Hirzebruch-Riemann-Roch theorem or [Mum70, Section 16]. Consequently, to determine the sign of $\chi(\mathscr{M})$, we merely have to understand the sign of the integrand in top degree $n$, that is, the sign of the coefficient at $x^{n}$ in the formal power series

$$
\left(\frac{-d x}{1-e^{d x}}\right)^{-1}\left(\frac{-d p x}{1-e^{d p x}}\right)\left(\frac{x}{1-e^{-x}}\right)^{n+1} e^{(d p-d-n-1+s) x} .
$$

This series can be rewritten as

$$
\left(\frac{-d x}{1-e^{d x}}\right)^{-1}\left(\frac{x}{1-e^{-x}}\right)^{n+1} e^{-(d+n+1-s) h} \cdot\left(\frac{d p x}{1-e^{-d p x}}\right),
$$

where the prime $p>0$ only enters through the last factor. We temporarily regard $p$ as another indeterminate and the above expression as an element $\sum \lambda_{i}(p) x^{i}$ in the ring $\mathbb{Q}[p][[x]]$. It appears difficult to find a closed formula for the polynomial $\lambda_{n}(p)$. However, it is possible to determine the sign of the leading coefficient.

Recall that the formal power series $x /\left(1-e^{-x}\right)=1+x / 2+\sum_{i=2}^{\infty} B_{i} x^{i} / i$ ! can be expressed in terms of the Bernoulli numbers $B_{i} \in \mathbb{Q}$. We follow the convention that $B_{1}=1 / 2$. Since $n \geqslant 2$, the last factor in (9.1) becomes

$$
1+\frac{d p}{2} x+\cdots+\frac{(d p)^{n-1} B_{n-1}}{(n-1) !} x^{n-1}+\frac{(d p)^{n} B_{n}}{n !} x^{n}+\cdots,
$$

while the product of the first three factors in (9.1) starts with

$$
\left(1-\frac{d x}{2}+\cdots\right)^{-1}\left(1+\frac{x}{2}+\cdots\right)^{n+1}\left(1-\frac{(d+n+1-s) x}{1 !}+\cdots\right) .
$$

This equals $1+\frac{1}{2}(2 s-d-n-1) x$ modulo $x^{2}$. The coefficient of $x^{n}$ in the power series (9.1) thus takes the form

$$
\lambda_{n}(p)=\frac{B_{n}(d p)^{n}}{n !}+\frac{(2 s-d-n-1) B_{n-1}(d p)^{n-1}}{2(n-1) !}+\text { lower degrees } .
$$

By assumption, $2 s-d-n-1>0$. The signs of the Bernoulli numbers are given by

$$
\operatorname{sign}\left(B_{2 j}\right)=(-1)^{j-1} \text { and } B_{2 j+1}=0,
$$

for every $j \geqslant 1$. The former can be deduced from the formula $B_{2 j}=\left((-1)^{j-1} 2(2 j) ! /(2 \pi)^{2 j}\right) \zeta(2 j)$ involving the Riemann zeta function $\zeta(z)$. It follows that the degree of $\lambda_{n}(p)$ depends on the parity of $n$. However, for both $n=2 j$ even and $n=2 j+1$ odd, the sign of the leading term in (9.2) equals $(-1)^{j-1}$. In other words, for $n \neq \equiv 2$ modulo 4 , this sign is always negative. Regarding the symbol $p$ again as a prime number, we conclude that $\lambda_{n}(p)<0$ for all sufficiently large primes $p \gg 0$, and then $\chi(\mathscr{M})<0$.

Proof for Theorem 9.1. Seeking a contradiction, we assume that there are infinitely many primes $p>0$ for which the Moret-Bailly family $Y$ lifts to the ring of truncated Witt vectors $W_{2}(k)$, 


\section{RÖSSLER AND S. SCHRÖER}

with fixed integers $n \geqslant 2$ and $d \geqslant 1$. Choose some $s>(n+d+1) / 2$. By Proposition 9.3, we find some sufficiently large $p \geqslant \operatorname{dim}(Y)$ such that there is an ample invertible sheaf $\mathscr{L}$ on $Y$ with $\chi\left(\mathscr{L} \otimes \omega_{Y}\right)<0$. Consequently, $h^{i}\left(\mathscr{L} \otimes \omega_{Y}\right)>0$ for some odd degree $i$. On the other hand, the Deligne-Illusie form of Kodaira-Akizuki-Nakano vanishing [DI87, Corollary 2.8] ensures that $h^{j}\left(\mathscr{L} \otimes \omega_{Y}\right)=0$ for all $j>0$, so we have a contradiction.

Of course, for fixed values of $n \geqslant 1$ one may compute the coefficient $\lambda_{n}(p)$ in the formal power series (9.1), either by hand or with computer algebra, as a polynomial in $d, p, s$. For $n=1$, we have $\lambda_{1}(p)=d(p-1) / 2+s-1$, which is always positive. For $n=3$ and $s=1$, we obtain

$$
\lambda_{3}(p)=-\frac{d^{3}+2 d^{2}}{24} p^{2}+\frac{d^{3}+3 d^{2}+2 d}{12} p-\frac{d^{3}+2 d^{2}+2 d}{24} .
$$

The zero of the derivative $\lambda_{3}^{\prime}(p)$ is the rational number $r=\left(d^{3}+3 d^{2}+2 d\right) /\left(d^{3}+2 d^{2}\right)$, which for all $d \geqslant 1$ satisfies $r<2$. Arguing as above, we obtain the following.

Proposition 9.4. For $n=3$ and $d \geqslant 1$, the Moret-Bailly family $Y$ over a perfect field of characteristic $p \geqslant 7=\operatorname{dim}(Y)$ does not lift to the ring of Witt vectors $W_{2}(k)$ of length two.

\section{ACKNOWLEDGEMENTS}

We wish to thank the referee for their careful reading and valuable suggestions, in particular for pointing out a mistake in the first version of the proof for Proposition 7.4. The second author is grateful for the hospitality during his visit at Pembroke College Oxford, where much of the work was carried out. We like to thank Laurent Moret-Bailly for useful remarks on the literature, and Emilian Zdanowicz for pointing out the results in [AZ17] and [Zda21]. Last but not least, we are grateful to Ludvig Olsson for his remarks and for his contribution to this paper (see the discussion in the introduction).

\section{REFERENCES}

Ach15 P. Achinger, A characterization of toric varieties in characteristic $p$, Int. Math. Res. Not. 2015 (2015), no. 16, 6879-6892; doi:10.1093/imrn/rnu151doi:10.1093/imrn/rnu151.

AGV73 M. Artin, A. Grothendieck, and J.L. Verdier (eds), Théorie des topos et cohomologie étale des schémas (SGA 4), Tome 3, Lecture Notes in Math., vol. 305 (Springer-Verlag, Berlin, 1973); doi: 10.1007/BFb0070714.

Art69 M. Artin, Algebraization of formal moduli. I, in Global Analysis (Papers in Honor of K. Kodaira) (eds D. Spencer and S. Iyanaga) (Univ. Tokyo Press, Tokyo, 1969), 21-71.

Ati56 M. Atiyah, On the Krull-Schmidt theorem with application to sheaves, Bull. Soc. Math. France 84 (1956), 307-317; doi: 10.24033/bsmf.1475.

AZ17 P. Achinger and M. Zdanowicz, Non-liftable Calabi-Yau varieties in characteristic $p \geqslant 5,2017$, arXiv: 1710.08202 .

BBM82 P. Berthelot, L. Breen, and W. Messing, Théorie de Dieudonné cristalline. II, Lecture Notes in Math., vol. 930 (Springer-Verlag, Berlin, 1982); doi:10.1007/BFb0093025.

BCFS10 W. Bosma, J. Cannon, C. Fieker, and A. Steel (eds), Handbook of Magma functions, Edition 2.16, Sydney, 2010.

Bea83 A. Beauville, Variétés Kähleriennes dont la première classe de Chern est nulle, J. Differential Geom. 18 (1983), no. 4, 755-782; doi:10.4310/jdg/1214438181. 


\section{Moret-BAILly FAMILIES AND NON-LIFTABLE SCHEMES}

BM04 J. Brillhart and P. Morton, Class numbers of quadratic fields, Hasse invariants of elliptic curves, and the supersingular polynomial, J. Number Theory 106 (2004), no. 1, 79-111; doi:10.1016/ j.jnt.2004.01.006.

Bog74 F.A. Bogomolov, On the decomposition of Kähler manifolds with a trivial canonical class, Math. USSR Sb. 22 (1974), no. 4, 580-583; doi:10.1070/SM1974v022n04ABEH001706.

Bog78_ , Hamiltonian Kählerian manifolds, Dokl. Akad. Nauk SSSR 243 (1978), no. 5, 11011104.

Bot57 R. Bott, Homogeneous vector bundles, Ann. of Math. 66 (1957), no. 2, 203-248; doi:10.2307/ 1969996.

Cao19 J. Cao, Albanese maps of projective manifolds with nef anticanonical bundles, Ann. Sci. Éc. Norm. Supér. (4) 52 (2019), no. 5, 1137-1154; doi:10.24033/asens.240.

CS12 S. Cynk and M. Schütt, Non-liftable Calabi-Yau spaces, Ark. Mat. 50 (2012), no. 1, 23-40; doi:10.1007/s11512-010-0130-4.

CvS09 S. Cynk and D. van Straten, Small resolutions and non-liftable Calabi-Yau threefolds, Manuscripta Math. 130 (2009), no. 2, 233-249; doi:10.1007/s00229-009-0293-0.

Del81 P. Deligne, Relèvement des surfaces K3 en caractéristique nulle, Algebraic Surfaces (Orsay, 1976-78) (eds J. Giraud, L. Illusie, and M. Raynaud), Lecture Notes in Math., vol. 868 (Springer, Berlin - New York, 1981), 58-79; doi:10.1007/BFb0090646.

DG70a M. Demazure and A. Grothendieck (eds), Schémas en groupes. III: Structure des schémas en groupes réductifs, Lecture Notes in Math., vol. 153 (Springer-Verlag, Berlin, 1970); doi:10.1007/ BFb0059027.

DG70b M. Demazure and P. Gabriel, Groupes algébriques (Masson, Paris, 1970).

DI87 P. Deligne and L. Illusie, Relèvements modulo $p^{2}$ et décomposition du complexe de de Rham, Invent. Math. 89 (1987), no. 2, 247-270; doi:10.1007/BF01389078.

Dol82 I. Dolgachev, Weighted projective varieties, Group Actions and Vector Fields (Vancouver, B.C., 1981) (J. Carrell, ed.), Lecture Notes in Math., vol. 956 (Springer, Berlin, 1982), 34-71; doi: 10.1007/BFb0101508.

Eke87 T. Ekedahl, Foliations and inseparable morphisms, Algebraic Geometry, Bowdoin, 1985 (Brunswick, Maine, 1985) (ed. S. Bloch), Proc. Sympos. Pure Math., vol. 46 (Amer. Math. Soc., Providence, RI, 1987), 139-149; doi:10.1090/pspum/046.2/927978.

EN62 J. A. Eagon and D. G. Northcott, Ideals defined by matrices and a certain complex associated with them, Proc. Roy. Soc. London Ser. A 269 (1962), 188-204; doi:10.1098/rspa.1962.0170.

ES05 T. Ekedahl and N. I. Shepherd-Barron, Tangent lifting of deformations in mixed characteristic, J. Algebra 291 (2005), no. 1, 108-128; doi:10.1016/j.jalgebra.2005.05.023.

FC90 G. Faltings and C.-L. Chai, Degeneration of abelian varieties, Ergeb. Math. Grenzgeb. (3), vol. 22 (Springer-Verlag, Berlin, 1990); doi:10.1007/978-3-662-02632-8.

Ful98 W. Fulton, Intersection theory, 2nd edn, Ergeb. Math. Grenzgeb. (3), vol. 2 (Springer-Verlag, Berlin, 1998); doi:10.1007/978-1-4612-1700-8.

GD60 A. Grothendieck and J. Dieudonné, Éléments de géométrie algébrique. I. Le langage des schémas, Publ. Math. Inst. Hautes Études Sci. 4 (1960); doi:10.1007/BF02684778.

GD61_ Éléments de géométrie algébrique. III. Étude cohomologique des faisceaux cohérents. I, Publ. Math. Inst. Hautes Études Sci. 11 (1961); doi:10.1007/BF02684274.

GD66 - Éléments de géométrie algébrique. IV. Étude locale des schémas et des morphismes de schémas III, Publ. Math. Inst. Hautes Études Sci. 28 (1966); doi:10.1007/BF02684343.

Gro57 A. Grothendieck, Sur quelques points d'algèbre homologique, Tohoku Math. J. 9 (1957), no. 2, 119-221; doi:10.2748/tmj/1178244839.

Gro61_, Techniques de construction et théorèmes d'existence en géométrie algébrique. IV. Les schémas de Hilbert, Sémin. Bourbaki, Vol. 1960/1961, Exp. No. 221, Astérisque 6 (1961), 249276. 


\section{RÖSSLER AND S. SCHRÖER}

Gro62

Technique de descente et théorèmes d'existence en géométrie algébrique. VI. Les schémas de Picard: propriétés générales, Sémin. Bourbaki, Vol. 1961/1962, Exp. No. 236, Astérisque 7 (1962), 221-243.

Gro66 _ Un théorème sur les homomorphismes de schémas abéliens, Invent. Math. 2 (1966), no. 1, 59-78; doi:10.1007/BF01403390.

Gro72 A. Grothendieck (ed.), Groupes de monodromie en géométrie algébrique (SGA 7 I), Lecture Notes in Math., vol. 288 (Springer-Verlag, Berlin, 1972); doi:10.1007/BFb0068688.

Hir99 M. Hirokado, A non-liftable Calabi-Yau threefold in characteristic 3, Tohoku Math. J. 51 (1999), no. 4, 479-487; doi:10.2748/tmj/1178224716.

HIS07 M. Hirokado, H. Ito, and N. Saito, Calabi-Yau threefolds arising from fiber products of rational quasi-elliptic surfaces. I, Ark. Mat. 45 (2007), no. 2, 279-296; doi:10.1007/s11512-0070041-1.

HIS08_— Calabi-Yau threefolds arising from fiber products of rational quasi-elliptic surfaces. II, Manuscripta Math. 125 (2008), no. 3, 325-343; doi:10.1007/s00229-007-0151-x.

Hua01 I.-C. Huang, Cohomology of projective space seen by residual complex, Trans. Amer. Math. Soc. 353 (2001), no. 8, 3097-3114; doi:10.1090/S0002-9947-01-02686-1.

Kat81 N. Katz, Serre-Tate local moduli, Algebraic Surfaces (Orsay, 1976-78) (eds J. Giraud, L. Illusie, and M. Raynaud), Lecture Notes in Math., vol. 868 (Springer, Berlin, 1981), 138-202; doi: 10.1007/BFb0090648.

Kaw92 Y. Kawamata, Unobstructed deformations. A remark on a paper of Z. Ran: "Deformations of manifolds with torsion or negative canonical bundle" [J. Algebraic Geom. 1 (1992), no. 2, 279-291], J. Algebraic Geom. 1 (1992), no. 2, 183-190.

KR03 K. Köhler and D. Roessler, A fixed point formula of Lefschetz type in Arakelov geometry. IV. The modular height of C.M. abelian varieties, J. reine angew. Math. 556 (2003), 127-148; doi: 10.1515/crl1.2003.017.

KS06 M. Kashiwara and P. Schapira, Categories and sheaves, Grundlehren Math. Wiss., vol. 332 (Springer-Verlag, Berlin, 2006); doi:10.1007/3-540-27950-4.

LS21 B. Laurent and S. Schröer, Para-abelian varieties and Albanese maps, 2021, arXiv:2101.10829.

Mor81 L. Moret-Bailly, Familles de courbes et de variétés abéliennes sur $\mathbb{P}^{1}$, Astérisque 86 (1981), 109-140.

Mor92 A. Moriwaki, A criterion of openness of a family of nef line bundles, Manuscripta Math. 75 (1992), no. 3, 327-331; doi:10.1007/BF02567088.

Mum70 D. Mumford, Abelian varieties, Tata Inst. Fund. Res. Stud. Math., vol. 5 (Oxford Univ. Press, London, 1970).

NO80 P. Norman and F. Oort, Moduli of abelian varieties, Ann. of Math. 112 (1980), no. 3, 413-439; doi:10.2307/1971152.

Oda69 T. Oda, The first de Rham cohomology group and Dieudonné modules, Ann. Sci. École Norm. Sup. (4) 2 (1969), no. 1, 63-135; doi:10.24033/asens.1175.

Ols16 M. Olsson, Algebraic spaces and stacks, Amer. Math. Soc. Colloq. Publ., vol. 62 (Amer. Math. Soc., Providence, RI, 2016); doi:10.1090/coll/062.

Oor75 F. Oort, Which abelian surfaces are products of elliptic curves?, Math. Ann. 214 (1975), 35-47; doi: 10.1007/BF01428253.

OSS80 C. Okonek, M. Schneider, and H. Spindler, Vector bundles on complex projective spaces, Progr. Math., vol. 3 (Birkhäuser, Boston, MA, 1980); doi:10.1007/978-1-4757-1460-9.

PZ19 Z. Patakfalvi and M. Zdanowicz, On the Beauville-Bogomolov decomposition in characteristic $p \geqslant 0,2019$, arXiv:1912.12742.

Ran92 Z. Ran, Deformations of manifolds with torsion or negative canonical bundle, J. Algebraic Geom. 1 (1992), no. 2, 279-291. 


\section{MoRet-BAILly FAMILIES AND NON-LIFTABLE SCHEMES}

Ray70 M. Raynaud, Faisceaux amples sur les schémas en groupes et les espaces homogènes, Lecture Notes in Math., vol. 119 (Springer-Verlag, Berlin - New York, 1970); doi : 10.1007/BFb0059504.

Rim80 D. S. Rim, Equivariant G-structure on versal deformations, Trans. Amer. Math. Soc. 257 (1980), no. 1, 217-226; doi:10.2307/1998132.

Sch09 C. Schoen, Desingularized fiber products of semi-stable elliptic surfaces with vanishing third Betti number, Compos. Math. 145 (2009), no. 1, 89-111; doi:10.1112/S0010437X08003801.

Sch03 S. Schröer, The $T^{1}$-lifting theorem in positive characteristic, J. Algebraic Geom. 12 (2003), no. 4, 699-714; doi:10.1090/S1056-3911-03-00330-8.

Sch04 Some Calabi-Yau threefolds with obstructed deformations over the Witt vectors, Compos. Math. 140 (2004), no. 6, 1579-1592; doi:10.1112/S0010437X04000545.

Ser60 J.-P. Serre, Universal morphisms and Albanese varieties, in Variétés de Picard, Sém. C. Chevalley (1960), no. 10.

Shi79 T. Shioda, Supersingular K3 surfaces, Algebraic Geometry (Proc. Summer Meeting, Univ. Copenhagen, Copenhagen, 1978) (ed. K. Lonsted), Lecture Notes in Math., vol. 732 (Springer, Berlin, 1979), 564-591; doi:10.1007/BFb0066664.

ST18 S. Schröer and Y. Takayama, On equivariant formal deformation theory, Rend. Circ. Mat. Palermo 67 (2018), no. 3, 409-419; doi:10.1007/s12215-017-0322-x.

Tat75 J. Tate, Algorithm for determining the type of a singular fiber in an elliptic pencil, Modular Functions of One Variable, IV (Proc. Internat. Summer School, Univ. Antwerp, Antwerp, 1972) (eds B. Birch and W. Kuyk), Lecture Notes in Math., vol. 476 (Springer, Berlin, 1975), 33-52; doi:10.1007/BFb0097582.

Tia87 G. Tian, Smoothness of the universal deformation space of compact Calabi-Yau manifolds and its Petersson-Weil metric, Mathematical Aspects of String Theory (San Diego, Calif., 1986) (ed. S. Yau), Adv. Ser. Math. Phys., vol. 1 (World Sci. Publishing, Singapore, 1987), 629-646; doi:10.1142/9789812798411_0029.

Tod80 A. N. Todorov, Applications of the Kähler-Einstein-Calabi-Yau metric to moduli of K3 surfaces, Invent. Math. 61 (1980), no. 3, 251-265; doi:10.1007/BF01390067.

Tot19 B. Totaro, The failure of Kodaira vanishing for Fano varieties, and terminal singularities that are not Cohen-Macaulay, J. Algebraic Geom. 28 (2019), no. 4, 751-771; doi:10.1090/jag/724.

TV13 M. Talpo and A. Vistoli, Deformation theory from the point of view of fibered categories, Handbook of Moduli, Vol. III (eds G. Farkas and I. Morrison), Adv. Lect. Math. (ALM), vol. 26 (Int. Press, Somerville, MA, 2013), 281-397.

Zda21 M.E. Zdanowicz, Arithmetically rigid schemes via deformation theory of equivariant vector bundles, Math. Z. 297 (2021), no. 1-2, 361-387; doi:10.1007/s00209-020-02513-9.

Damian Rössler rossler@maths.ox.ac.uk

Mathematical Institute, University of Oxford, Andrew Wiles Building, Rad- cliffe Observatory Quarter, Woodstock Road, Oxford, OX2 6GG, United Kingdom

Stefan Schröer schroeer@math.uni-duesseldorf.de

Mathematisches Institut, Heinrich-Heine-Universität, 40204 Düsseldorf, Germany 